2012

\title{
Psr J0737-3039B: A Probe Of Radio Pulsar Emission Heights
}

B. B. P. Perera

D. Lomiashvili

K. N. Gourgouliatos

M.A. McLaughlin

M. Lyutikov

Follow this and additional works at: https://researchrepository.wvu.edu/faculty_publications

\section{Digital Commons Citation}

Perera, B. B. P.; Lomiashvili, D.; Gourgouliatos, K. N.; McLaughlin, M. A.; and Lyutikov, M., "Psr J0737-3039B: A Probe Of Radio Pulsar Emission Heights" (2012). Faculty Scholarship. 642.

https://researchrepository.wvu.edu/faculty_publications/642 


\title{
PSR J0737-3039B: A PROBE OF RADIO PULSAR EMISSION HEIGHTS
}

\author{
B. B. P. Perera ${ }^{1}$, D. Lomiashvili ${ }^{2}$, K. N. Gourgouliatos ${ }^{2}$, M. A. McLaughlin ${ }^{1,3}$, and M. Lyutikov ${ }^{2}$ \\ ${ }^{1}$ Department of Physics, West Virginia University, Morgantown, WV 26506, USA \\ ${ }^{2}$ Department of Physics, Purdue University, West Lafayette, IN 47907, USA \\ Received 2011 October 12; accepted 2012 March 3; published 2012 April 24
}

\begin{abstract}
In the double pulsar system PSR J0737-3039A/B, the strong wind produced by pulsar A distorts the magnetosphere of pulsar B. The influence of these distortions on the orbital-dependent emission properties of pulsar B can be used to determine the location of the coherent radio emission generation region in the pulsar magnetosphere. Using a model of the wind-distorted magnetosphere of pulsar B and the well-defined geometrical parameters of the system, we determine the minimum emission height to be $\sim 20 R_{\mathrm{NS}}$ in the two bright orbital longitude regions. We can determine the maximum emission height by accounting for the amount of deflection of the polar field line with respect to the magnetic axis using the analytical magnetic reconnection model of Dungey and the semi-empirical numerical model of Tsyganenko. Both of these models estimate the maximum emission height to be $\sim 2500 R_{\mathrm{NS}}$. The minimum and maximum emission heights we calculate are consistent with those estimated for normal isolated pulsars.
\end{abstract}

Key words: pulsars: individual (PSR J0737-3039A/B) - stars: neutron

Online-only material: color figures

\section{INTRODUCTION}

The mechanism of pulsar radio emission and its origin within the pulsar magnetosphere are not well understood (see, e.g., Hankins et al. 2009). In general, it is thought to be due to coherent radiation from relativistic plasma streaming along open magnetic field lines. Radio emission height estimates can constrain the emission mechanism to some extent. In normal isolated pulsars, radio emission heights have been estimated from their emission geometry inferred from radio polarization combined with the rotating vector model (Radhakrishnan \& Cooke 1969) and the pulse profile widths (Gil \& Kijak 1993; Kijak \& Gil 1997; Kijak \& Gil 2003). Gangadhara \& Gupta (2001) and Dyks et al. (2004) have also proposed a phaseshift method to determine the emission height. In general, these methods show that core component emission originates very close to the surface of the neutron star (NS), but the conal components come from well above the surface (Rankin 1990; Mitra \& Rankin 2002). However, these techniques are limited in that we observe only a small section of the magnetosphere of these isolated pulsars due to an unchanging line of sight.

PSR J0737-3039A/B is a unique binary system that provides an excellent opportunity to study different emission regions due to relativistic spin precession, allowing us to observe different portions of the magnetosphere. We can also explore magnetospheric distortion, which affects the observed emission pattern. The two NSs of this system orbit each other in a $2.4 \mathrm{hr}$ orbit; this is the only known pulsar binary system in which both NSs have been detectable as radio pulsars (Burgay et al. 2003; Lyne et al. 2004). The first-born recycled pulsar-hereafter A - has a spin period of $23 \mathrm{~ms}$ and the secondborn pulsar-hereafter B-has a spin period of $2.8 \mathrm{~s}$. The pulse profile of A has been stable since its discovery, but that of B has dramatically evolved through five years of observation, culminating in its radio disappearance in 2008 March (Perera et al. 2010).

\footnotetext{
3 Also an adjunct astronomer at the National Radio Astronomy Observatory, Green Bank, WV 24944, USA.
}

Due to the unstable features of B's pulse profiles, both on long timescales and within a single orbit, it is challenging to understand the emission geometry and the emission mechanism. By fitting a model to eclipses of $\mathrm{A}$ due to absorption in the magnetosphere of B, Breton et al. (2008) constrained the geometrical parameters to be $\alpha=70.9(4)^{\circ}$ and $\theta=130.0(4)^{\circ} .4$ Here, $\alpha$ is the misalignment of the magnetic axis with respect to the spin axis and $\theta$ is the colatitude of the spin axis. Unfortunately, B shows very little radio polarization, making it impossible to constrain the geometry from polarization measurements (Demorest et al. 2004). Breton et al. (2008) also constrained the precessional phase of the spin axis, measured from our line of sight, to be $\phi_{\text {prec }}=51.2(8)^{\circ}$ at an epoch of 2006 May 2 (i.e., MJD 53857) and found that this phase is changing with time at a rate of $4.8(7)^{\circ} \mathrm{yr}^{-1}$. This is consistent with the rate of $5.061(2)^{\circ} \mathrm{yr}^{-1}$ predicted by general relativity (Barker \& O'Connell 1975). Recently, Perera et al. (2010) analyzed the pulse profile evolution of $\mathrm{B}$ and independently determined the above angles using a simple model based on geodetic spin precession as proposed by Clifton \& Weisberg (2008). According to this model, the beam must be elliptical and horse-shoe shaped in order to explain the observed singleto double-peak pulse profile evolution and the disappearance of radio emission. The estimations of the above angles in this model are consistent with those predicted by Breton et al. (2008), within the $2 \sigma$ errors. From these studies, we believe that the geometrical parameters of B are well known. We can therefore use these values in this paper to determine the emission geometry.

In this paper, we also explore the distortion of the magnetosphere of B. The almost edge-on orbital plane of the system, with inclination angle of 88.7 (Kramer et al. 2006), allows us to observe the eclipses of A with a duration of about $30 \mathrm{~s}$. By considering the relative transverse velocities of the two pulsars, $660 \mathrm{~km} \mathrm{~s}^{-1}$ (Lyne et al. 2004), the estimated size of B's magnetosphere is about $10 \%$ of its light cylinder radius of $\sim 1.3 \times 10^{10} \mathrm{~cm}$.

\footnotetext{
4 Here, and throughout the paper, the number in parentheses is the $1 \sigma$ uncertainty in the last quoted digit.
} 
This implies that the wind of A compresses the magnetosphere of B and disturbs its polar cap (Lyutikov 2004). This is due to the small separation of the pulsars $\left(\sim 9 \times 10^{10} \mathrm{~cm}\right.$ or $\left.2.9 \mathrm{lt}-\mathrm{s}\right)$ and the large spin-down luminosity of $\mathrm{A}\left(5.8 \times 10^{33} \mathrm{ergs}^{-1}\right)$ compared with $\mathrm{B}\left(1.6 \times 10^{30} \mathrm{ergs}^{-1}\right)$. This is analogous to the distortion of Earth's magnetosphere due to the solar wind. This interaction also results in an orbital modulation of $\mathrm{B}$; we detect bright emission from the pulsar only in two orbital phase regions of $185^{\circ}-235^{\circ}$ (hereafter BP1) and $265^{\circ}-305^{\circ}$ (hereafter BP2), and detect weak emission at phases of $340^{\circ}-30^{\circ}$ and $80^{\circ}-130^{\circ}$ (here, and throughout the paper, orbital phases are measured from the ascending node). Lyutikov (2005) claims that the pulsar has the same intrinsic radio intensity throughout the orbit and that the orbital modulation is due to the deflection of the magnetic polar field line with respect to the line of sight because of the influence of $\mathrm{A}$.

The wind interaction with the magnetosphere of $\mathrm{B}$ produces a bow shock between A and B; this is likely the boundary of the magnetosphere of $\mathrm{B}$. The shape of this boundary depends on the orientation of the magnetic axis of B. Lyutikov (2004) constrained the stand-off distance, or the distance from $\mathrm{B}$ to the vertex of the bow shock, to be $3.5 \times 10^{9} \mathrm{~cm}$ if the bow shock interface is a perfect resistor and $4 \times 10^{9} \mathrm{~cm}$ if it is partially resistive. These estimates inferred that the magnetosphere of $\mathrm{B}$ is located deep within its light cylinder and the open and closed field lines have a more complicated structure than that of an isolated pulsar. Since the wind-interaction boundary model is very important to study the emission geometry of $\mathrm{B}$, we derive it again in this paper with some improvements. This model describes the shape of the boundary for any orientation of the magnetic axis and allows us to model the open and closed field line structures more accurately.

This results in a method to use the derived field line structure to estimate the radio emission heights of B. Since the bow shock boundary is located deep inside the light cylinder, the correction due to rotation on the static dipole field, which introduced in retarded dipole field, is small (for retarded dipole field, refer Appendix A of Dyks \& Harding 2004). Therefore we assume non-rotating dipole field throughout the model. In our method, we assume that the emission comes from the direction tangential to the local field lines. We also assume that the emission comes from above the polar cap region, consistent with the narrow single- and double-peaked radio profiles.

The distortion of Earth's magnetosphere due to the solar wind has been studied using a large number of satellite observations and these data have been extensively modeled. Since the wind of A distorts B's magnetosphere in the same way that the solar wind does Earth's, some models for Earth's magnetic field line structure can be used to study the distortion of the magnetosphere of $\mathrm{B}$ and to determine the regions of radio emission. We use the Dungey (1961) planetary magnetosphere model and the Tsyganenko (2002a, 2002b) Earth magnetosphere models to set an upper limit on the radio emission height of $\mathrm{B}$.

We present our observational data in Section 2 and discuss observed mean pulse profiles. In Section 3, we explain the boundary model that describes the shape of the bow shock. Then we trace the dipole field lines and transform them from the corotating frame of the NS to the orbit-fixed frame. In order to derive the required angular radius of the elliptical beam for the emission height calculation, we re-analyze the beam with a different geometrical framework in Section 4. In Section 5, we present the method that we use to estimate the minimum emission height and our results. In Section 6, we describe the maximum emission height calculation using two different magnetosphere models. Finally in Section 7, we discuss our results and compare them with predicted emission heights for other pulsars. We also discuss height estimations from other methods, concluding that these are not applicable to pulsar B.

\section{OBSERVATIONS AND PULSE PROFILES}

We observed J0737-3039B with the 100 m Green Bank Telescope (GBT) in West Virginia since 2003 December 24 at multiple frequencies. Since $820 \mathrm{MHz}$ is the most common, we use only those data in this analysis. This is the same data set that we reported in Perera et al. (2010). However, we use better time resolution pulse profiles in this analysis compared with the previous paper. The data were taken using the GBT spectrometer SPIGOT with sampling time of $81.92 \mu$ s until 2009 January. After 2009 January, the spectrometer GUPPI was used with a sampling time of $61.44 \mu \mathrm{s}$. All the data were dedispersed and folded using the pulsar analysis package SIGPROC, assuming a dispersion measure of $48.914 \mathrm{~cm}^{-3}$ pc (Lyne et al. 2004). The ephemeris of Lyne et al. (2004) was used until 2006 and since then we have used the ephemeris of Kramer et al. (2006) to form mean pulse profiles.

The mean pulse profiles for BP1 are shown in Figure 1. We aligned the peak of the profiles to the pulse phase of 0.5 at each epoch. Note that these pulse profiles have a better resolution than those in Perera et al. (2010). The second peak of the pulse profile can be hidden with the low time resolution. Therefore, we use 1024 bins across the full pulse phase, resulting in an effective time resolution of $0.003 \mathrm{~s}$ in this analysis compared with $0.01 \mathrm{~s}$ in Perera et al. (2010). For example, the second peak of the pulse profile of MJD 53860 around pulse phase 0.52 in Figure 1 cannot be clearly seen in Figure 1 of Perera et al. (2010) on the same day with low time resolution. We use the pulse profiles from 23 days in Section 4, including 16 epochs in Figure 1, in order to derive the beam shape. We include some low signal-tonoise data (e.g., MJD 53481) in this particular analysis because the second peak became apparent around those days. Because these profiles appeared as single peaked, we ignored them in the geometrical modeling of the previous lower time resolution study. As in Perera et al. (2010), we fit one and two Gaussians for each single- and double-peaked pulse profile, respectively, and then calculate profile widths at different intensity levels. We use these data in Section 4 to determine the geometry of the pulsar and the beam.

\section{BOUNDARY MODEL}

Due to the distortion of the magnetosphere, the properties of pulsar B are different from those of normal isolated pulsars. In isolated pulsars, we can determine the size of the magnetosphere by modeling the open and closed field lines, given the size of their light cylinder. However, as mentioned earlier, the magnetosphere of B is located deep inside the light cylinder and the structure of the open and closed field lines is more complicated due to the distortion from A's wind.

In the first step, we approximate the structure of the magnetosphere as a rotating vacuum dipole. Then we apply a simple model for the wind-magnetosphere interaction, as in Lyutikov (2004). The wind of A creates a dynamic pressure on the magnetosphere of $\mathrm{B}$. The magnetosphere of $\mathrm{B}$ creates a magnetic pressure that opposes the wind pressure of A. At some point, these two pressures equal each other; this interface is likely the boundary of the magnetosphere of $\mathrm{B}$. This boundary can be used 


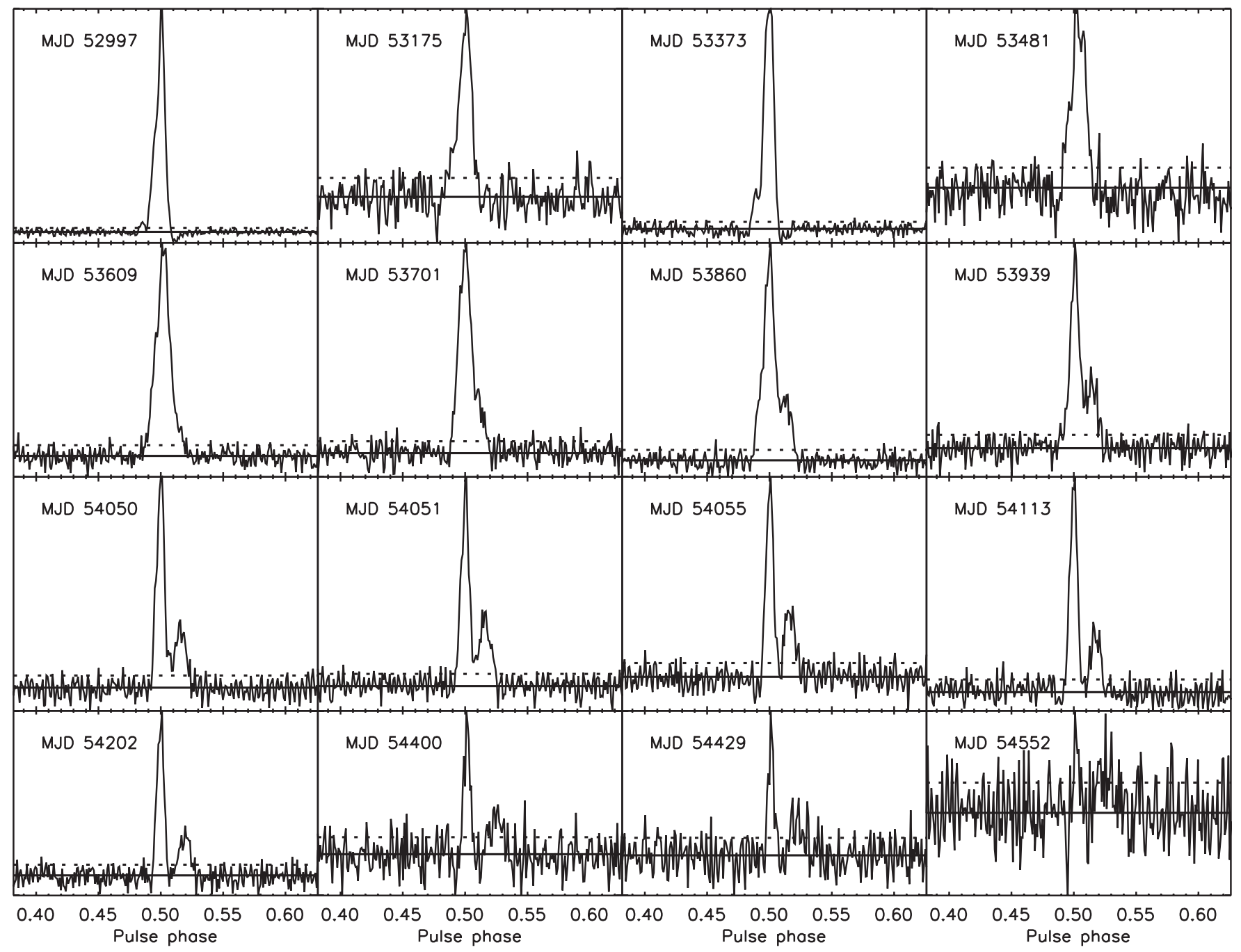

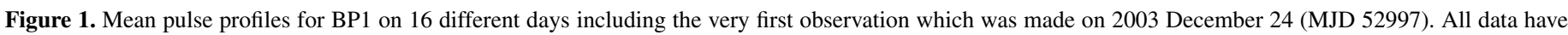

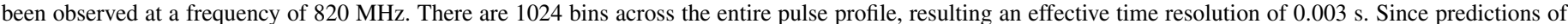

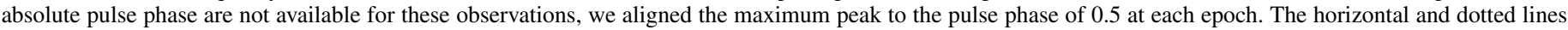

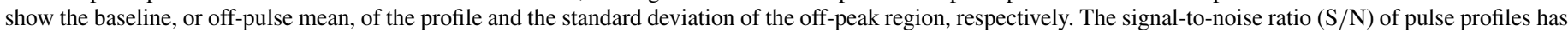
decreased significantly from 284 (on MJD 52997) to 11 (on MJD 54552).

to calculate the last open and closed field lines. We derive an expression for this boundary by equating the two pressures,

$$
\vec{B}^{2}\left(\overrightarrow{r_{\mathrm{B}}}\right) /(8 \pi)=L_{\mathrm{A}} \cos ^{2}(\gamma) / 4 \pi c{\overrightarrow{r_{\mathrm{A}}}}^{2},
$$

where $\overrightarrow{r_{\mathrm{B}}}$ is the distance vector of the boundary with respect to pulsar $\mathrm{B}, \vec{B}\left(\overrightarrow{r_{\mathrm{B}}}\right)$ is the magnetic field of pulsar $\mathrm{B}$ at $\overrightarrow{r_{\mathrm{B}}}, L_{\mathrm{A}}$ is the spin-down luminosity of $\mathrm{A}, \overrightarrow{r_{\mathrm{A}}}$ is the distance vector of the boundary with respect to $\mathrm{A}$, and $\gamma$ is the angle between the normal to the boundary and $\overrightarrow{r_{A}}$ (see Figure 2 ). The relative pressures lead to a boundary much closer to B. For that reason we simplify the problem by setting the distance of the boundary with respect to A equal to the distance between the two pulsars and $\gamma$ equal to the angle between the normal to the boundary and the line connecting two pulsars. By assuming a magnetic dipole at the center of the coordinate system, we can write the magnetic field strength of the NS as $\vec{B}\left(\overrightarrow{r_{\mathrm{B}}}\right)=\left(3 \hat{r}_{\mathrm{B}}\left(\vec{m} \cdot \hat{r}_{\mathrm{B}}\right)-\vec{m}\right) / r_{\mathrm{B}}{ }^{3}$, where $\vec{m}=m(\cos \delta \cos \Omega t, \cos \delta \sin \Omega t, \sin \delta)$ is the magnetic moment, $\Omega$ is the rotational angular frequency, and $\delta$ is the angle between the magnetic moment and the line connecting the two pulsars (see Figure 2).
In isolated pulsars, we believe that the spin-down is caused by the power carried out along the magnetic field lines that are open with respect to the light cylinder (Contopoulos \& Spitkovsky 2006). These open magnetic field lines start from the polar cap region of the NS surface and their shape changes with respect to the magnetic inclination. Spitkovsky (2006) proposed a realistic form of the spin-down luminosity of an isolated pulsar as a function of the magnetic inclination angle. However, the open field line structure of pulsar B is somewhat different than that for an isolated pulsar and, therefore, we need to define the magnetic field lines with respect to the bow shock boundary. Therefore, we modified the spin-down luminosity equation that is given in Spitkovsky (2006) by including the area of the polar cap region. This can be written as

$$
L_{\mathrm{B}}=\Omega^{2} B_{0}^{2} S^{2}\left(1+\sin ^{2} \alpha\right) / 4 \pi^{2} c,
$$

where $\Omega$ is the rotational angular frequency, $B_{0}$ is the polar magnetic field of pulsar B, $S$ is the area of the polar cap region, and $\alpha$ is the magnetic inclination. As we mentioned earlier, with previous geometry models (Breton et al. 2008; Perera et al. 2010), $\alpha$ is taken to be $\sim 70^{\circ}$. Note that the original version of 


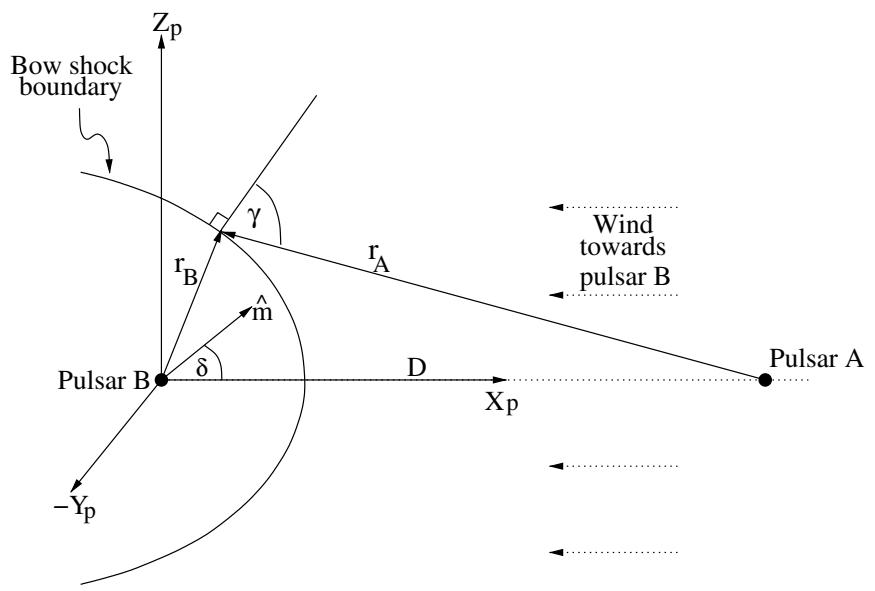

Figure 2. Geometry of the wind-magnetosphere interaction model. Pulsar A is located at a distance $D\left(\sim 9 \times 10^{10} \mathrm{~cm}\right)$ away from $\mathrm{B}$ along the $x_{\mathrm{p}}$-axis. The wind of $\mathrm{A}$ comes along the $-x_{\mathrm{p}}$ direction and is shocked near $\mathrm{B}$. The physical interface has the shape of a bow shock and this is the boundary of the magnetosphere of $\mathrm{B} . \overrightarrow{\mathrm{B}}$ is the position vector at any point on the interface with respect to $\mathrm{B} . \overrightarrow{r_{\mathrm{A}}}$ is the position vector of this point with respect to $\mathrm{A}$ and $\gamma$ is the angle between the normal to the boundary at this point and $\overrightarrow{r_{\mathrm{A}}} . \delta$ is the angle between the magnetic moment axis $\hat{m}$ and the line connecting the two pulsars.

the equation (as in Spitkovsky 2006) can be obtained by taking $S$ to be the area of the polar cap of a dipole as defined by the open magnetic field lines with respect to the light cylinder.

In order to determine the boundary, we solve Equation (1) numerically and then use Equation (2) to determine the value of the magnetic field. To do so, we simplified the problem to a twodimensional form in which the bow shock is represented by an equation involving $x_{\mathrm{p}}$ and $z_{\mathrm{p}}$ and lies on that plane. The equation of the bow shock then has the form $G\left(x_{\mathrm{p}}, z_{\mathrm{p}}\right)=f\left(z_{\mathrm{p}}\right)-x_{\mathrm{p}}$, which must be solved in order to determine the shape of the bow shock. Let the radial vector $\overrightarrow{r_{\mathrm{B}}}=f\left(z_{\mathrm{p}}\right) \hat{x}_{\mathrm{p}}+z \hat{z}_{\mathrm{p}}$ and the vector normal to the boundary $\vec{n}=\vec{\nabla} \cdot G\left(x_{\mathrm{p}}, z_{\mathrm{p}}\right)$. Then the dot product of these two gives the angle $\cos ^{2}(\gamma)=1 /\left(1+\left(d f / d z_{\mathrm{p}}\right)^{2}\right)$ and this can be substituted in Equation (1). According to our two-dimensional form, we can write the magnetic moment $\vec{m}=m \cos \delta \hat{x_{\mathrm{p}}}+m \sin \delta \hat{z_{\mathrm{p}}}$ and then derive the magnetic field of $\mathrm{B}$ at distance $\overrightarrow{r_{\mathrm{B}}}, \vec{B}\left(\overrightarrow{r_{\mathrm{B}}}\right)$, as a function of $m, f\left(z_{\mathrm{p}}\right), z$, and $\delta$. Then Equation (1) reduces to a first-order differential equation of $f\left(z_{\mathrm{p}}\right)$ and the solution determines the shape of the bow shock. First we assume an initial value for the magnetic moment, $m$, of $\mathrm{B}$ with a possible magnetic orientation, $\delta$, and solve the problem to determine the shape of the bow shock. Then we find which are the last closed field lines defined with respect to the bow shock and finally the shape and the area of the polar cap.

We repeat the procedure for 16 values of the angle $\delta$ between the magnetic moment and the line connecting the two pulsars, evenly spaced between 0 and $\pi / 2$, and we find the area of the polar cap for each of those orientations. In the estimation of the average area of the polar cap, we have weighted appropriately the fact that some values of $\delta$ occur more frequently than others during an orbital period. Using the spin-down luminosity given in Equation (2) with the timing-derived $L_{\mathrm{B}}$, we find a new value for the magnetic field. We repeat this process with this new magnetic field until the value of the magnetic field converges. This happens after five to ten iterations for an initial guess of the magnetic field within a couple of orders of magnitude away from the convergence value. In order to represent the threedimensional version of the bow shock, we assume that it is axially symmetric around $x_{\mathrm{p}}$.
According to the best solution, the magnetic field of $B$ is constrained to be $B_{\mathrm{B}}=6.4 \times 10^{11} \mathrm{G}$, which is about a factor of two lower than the timing-derived value $1.2 \times 10^{12} \mathrm{G}$ assuming a vacuum dipole with a magnetic inclination of $90^{\circ}$ (Lyne et al. 2004). This new estimate is more realistic as it accounts for the boundary of the magnetosphere as the bow shock and a realistic magnetic inclination. The stand-off distance is constrained to be either $3.8 \times 10^{9} \mathrm{~cm}$ or $4.5 \times 10^{9} \mathrm{~cm}$ for the cases when the magnetic axis is either normal or parallel to the line connecting the two pulsars, respectively. Thus, the size of the boundary depends on the orientation of the magnetic axis of pulsar B. Moreover, the shape of the bow shock depends on the orientation of the magnetic axis. The stand-off distance corresponds approximately to $1 / 3$ of the light cylinder, thus for these distances the relativistic modifications are minimal and do not change the value of the stand-off distance by more than a few percent. For that reason we have chosen to calculate it using a vacuum dipole model rather than a more complicated geometry that takes into account relativistic effects as in Deutsch (1955). For simplicity, we assume that the boundary is axially symmetric around the vector connecting the two pulsars. A maximum deviation of roughly $20 \%$ of the actual shape from the symmetric case occurs when the angle $\delta$ is $90^{\circ}$. Therefore, the shape of the boundary is sensitive to an angle of $\delta$, having a range of $\left[0^{\circ}, 90^{\circ}\right]$. If the angle $\delta$ is greater than $90^{\circ}$, then the boundary considered the effective $\delta$ of $180^{\circ}-\delta$. For example, if $\delta$ is $100^{\circ}$, then the effective $\delta$ for the boundary shape is $80^{\circ}$. Therefore, with the assumption that the magnetic axis is nearly aligned with the line of sight at the radio emission detection, the effective angle $\delta$ is small $\left(\sim 5^{\circ}-35^{\circ}\right)$ in the orbital longitude region of BP2. Thus, the deviation of the boundary model from the actual geometry of the boundary is small and the assumption of a symmetric geometry is reasonable. However, the deviation in the orbital phase region of BP1 is significant due to large effective angles of $\delta\left(\sim 35^{\circ}-85^{\circ}\right)$.

To derive an expression for the physical shape of the boundary, we examine different shapes that fit our results. As a preliminary fit, a parabola is a good guess, but a fourth-order polynomial describes the boundary better, yielding the minimum chi-squared value when we fit to our results. The coefficients of this polynomial describe the variation and are functions of the angle $\delta$. The best-fit polynomial is

$$
x_{\mathrm{p}}=a(\delta)+b(\delta)\left(y_{\mathrm{p}}^{2}+z_{\mathrm{p}}^{2}\right)+c(\delta)\left(y_{\mathrm{p}}^{2}+z_{\mathrm{p}}^{2}\right)^{2},
$$

with a $\mathrm{B}$-centered coordinate system in which the $x_{\mathrm{p}}$-axis is toward $\mathrm{A}$, the $z_{\mathrm{p}}$-axis is normal to the orbital plane, and the $y_{\mathrm{p}}$-axis completes the right-handed coordinate system (see Figure 2). The three axes have units in centimeters. The coefficients $a(\delta), b(\delta)$, and $c(\delta)$ are

$$
\begin{aligned}
& a(\delta)=\left(\frac{0.83-0.01 \delta-0.06 \delta^{2}-0.05 \delta^{3}+0.03 \delta^{4}}{1.83 \times 10^{-10}}\right) \\
& b(\delta)=\left(\frac{-0.46+0.04 \delta-1.43 \delta^{2}+1.96 \delta^{3}-0.64 \delta^{4}}{5.45 \times 10^{9}}\right) \\
& c(\delta)=\left(\frac{-0.48-0.03 \delta+2.15 \delta^{2}-2.47 \delta^{3}+0.74 \delta^{4}}{1.62 \times 10^{29}}\right),
\end{aligned}
$$

where the angle $\delta$ is in radians and having a range of $[0, \pi / 2]$. Since they are functions of $\delta$, the boundary changes slightly with spin and orbital motions, as well as over time due to precession.

This boundary model is valid only up to $5 \times 10^{9} \mathrm{~cm}$ or $\sim 40 \%$ of the light cylinder radius, from B. Beyond this limit, 


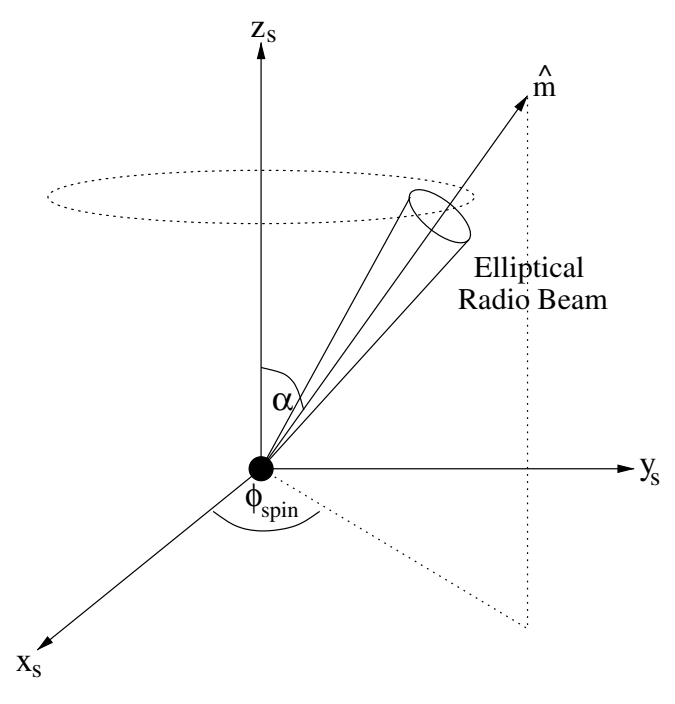

(a)

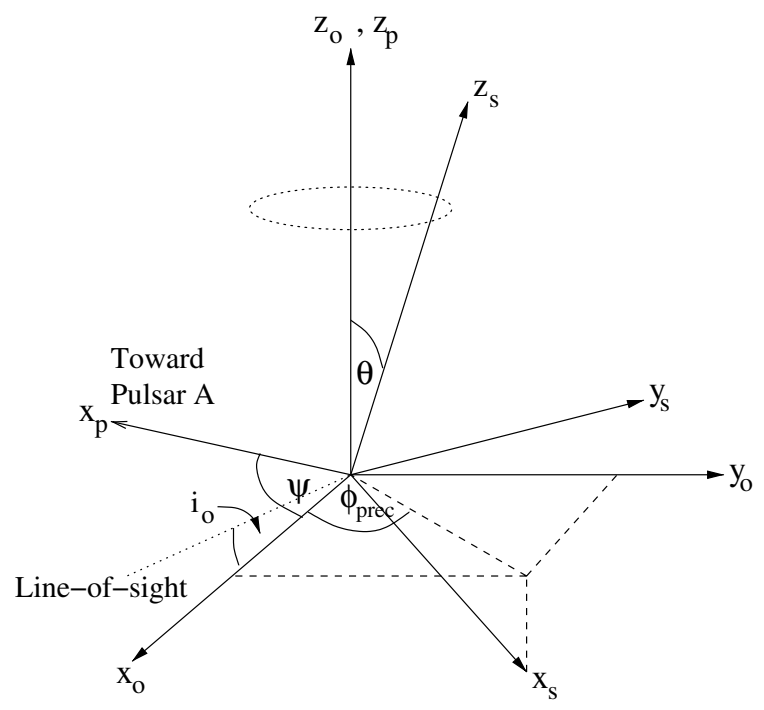

(b)

Figure 3. Cartesian coordinate systems that transform magnetic field lines from the corotating frame of the neutron star to the orbit-fixed frame. (a) The spin phase $\phi_{\text {spin }}$ and the misalignment of the magnetic axis $\alpha$ are defined in the frame $\left(x_{\mathrm{s}}, y_{\mathrm{s}}, z_{\mathrm{s}}\right)$. The spin phase is measured from the $x_{\mathrm{s}}$-axis. Therefore $\phi_{\text {spin }}=0$ is defined when the magnetic axis is in the $x_{\mathrm{s}}-z_{\mathrm{s}}$ plane. (b) The colatitude of the spin $\theta$ and the precessional phase $\phi_{\text {prec }}$ is defined in the frame $\left(x_{\mathrm{o}}, y_{\mathrm{o}}, z_{\mathrm{o}}\right)$. In this frame, the $x_{\mathrm{o}}$-axis is in the plane of the $z_{\mathrm{o}}$-axis and our line of sight (LOS). The spin precession $\phi_{\text {prec }}$ is measured from the $x_{0}$-axis. The frame of the bow shock boundary $\left(x_{\mathrm{p}}, y_{\mathrm{p}}, z_{\mathrm{p}}\right)$ is then placed in this orbit-fixed frame with $z_{\mathrm{p}} \| z_{\mathrm{o}}$ and rotate with an angle of $\psi$ defined from the $x_{\mathrm{o}}$-axis. Then the orbital phase is defined $\phi_{\mathrm{orb}}=\psi+90^{\circ}$ as it measures from the ascending node. $i_{\mathrm{o}}=90^{\circ}-i$, where $i$ is the orbital inclination.

the physical assumption of the dynamical pressure is incorrect because the wind pressure on the magnetic field should be zero when it is parallel to the boundary at large distances. Also we have assumed an undistorted magnetic field of B in the model and the distortions at large distances will be significant.

In summary, we determined the boundary of pulsar B by assuming equilibrium between the dynamical pressure of the wind of $\mathrm{A}$ and the magnetic pressure of the field of $\mathrm{B}$. The physical shape of the boundary is a bow shock and mathematically we can represent it as a fourth-order polynomial. Moreover, this shape depends on the orientation of the magnetic moment axis with respect to pulsar A. Thus, the coefficients of the best-fit polynomial depend on this orientation.

\subsection{Tracing the Dipole Field Lines}

Due to the wind interaction with the magnetosphere of $\mathrm{B}$, it is complicated to understand the structure of the open and closed field lines. To determine the polar cap region that is required for the emission height estimation, we calculate the last closed field lines by tracing them referring to the derived boundary model.

As is standard, we treat the magnetosphere of $B$ as a magnetic dipole. For an isolated pulsar, the last closed magnetic field lines are defined as those that just touch the light cylinder and the ones interior to the last closed field lines are considered open field lines. In our case the boundary is not the light cylinder but the bow shock, with the last closed field lines defined as those that just touch this bow shock. The polar cap region is defined by these particular field lines and the shape of it can be determined by the locations where these field lines cross the NS surface. Defining the polar cap is important since we think that the radio emission is produced above this region.

Unlike those of isolated pulsars, the magnetosphere of B is not symmetric around the magnetic axis due to the shape of the boundary. This can be clearly seen by tracing the last closed field lines. In order to trace the field lines, we use the dipole field line equation in polar coordinates

$$
\begin{aligned}
& r=r_{0} \sin ^{2}(\lambda) \\
& \phi=\phi_{0},
\end{aligned}
$$

where $r$ is the radial distance to a given point along the field line and $r_{0}$ is the field-line constant, or equatorial distance of the field line from the magnetic axis. The angle $\lambda$ is the colatitude of a given point along the field line and $\phi_{0}$ is the azimuth angle, or the longitude of the given field line. Then the Cartesian components of a particular field line are written as

$$
\begin{aligned}
& x=r \sin (\lambda) \cos (\phi) \\
& y=r \sin (\lambda) \sin (\phi) \\
& z=r \cos (\lambda),
\end{aligned}
$$

where the $z$-axis of the coordinate system is aligned with the magnetic moment axis and the other two axes are corotating with the NS.

To include the misalignment of the magnetic axis and also account for the spin phase, we transform Equation (6) to another frame where the $z$-axis is aligned with the spin axis. In this frame (see Figure 3(a)), the Cartesian components are

$$
\begin{aligned}
& x_{\mathrm{s}}=x \cos \alpha \cos \phi_{\mathrm{spin}}-y \sin \phi_{\mathrm{spin}}+z \sin \alpha \cos \phi_{\mathrm{spin}} \\
& y_{\mathrm{s}}=x \cos \alpha \sin \phi_{\mathrm{spin}}+y \cos \phi_{\mathrm{spin}}+z \sin \alpha \sin \phi_{\mathrm{spin}} \\
& z_{\mathrm{s}}=z \cos \alpha-x \sin \alpha,
\end{aligned}
$$

where $\alpha$ is the angle between the magnetic axis and the spin axis and $\phi_{\text {spin }}$ is the spin phase. We measure the spin phase from the $x_{\mathrm{s}}$-axis, which means that it is zero when the magnetic axis is in the $x_{\mathrm{s}}-z_{\mathrm{s}}$ plane.

The spin axis is also associated with the colatitude angle and the spin precessional phase. Geodetic spin precession changes the orientation of the spin axis with respect to our line of sight. In order to include the colatitude of the spin axis and effects of 

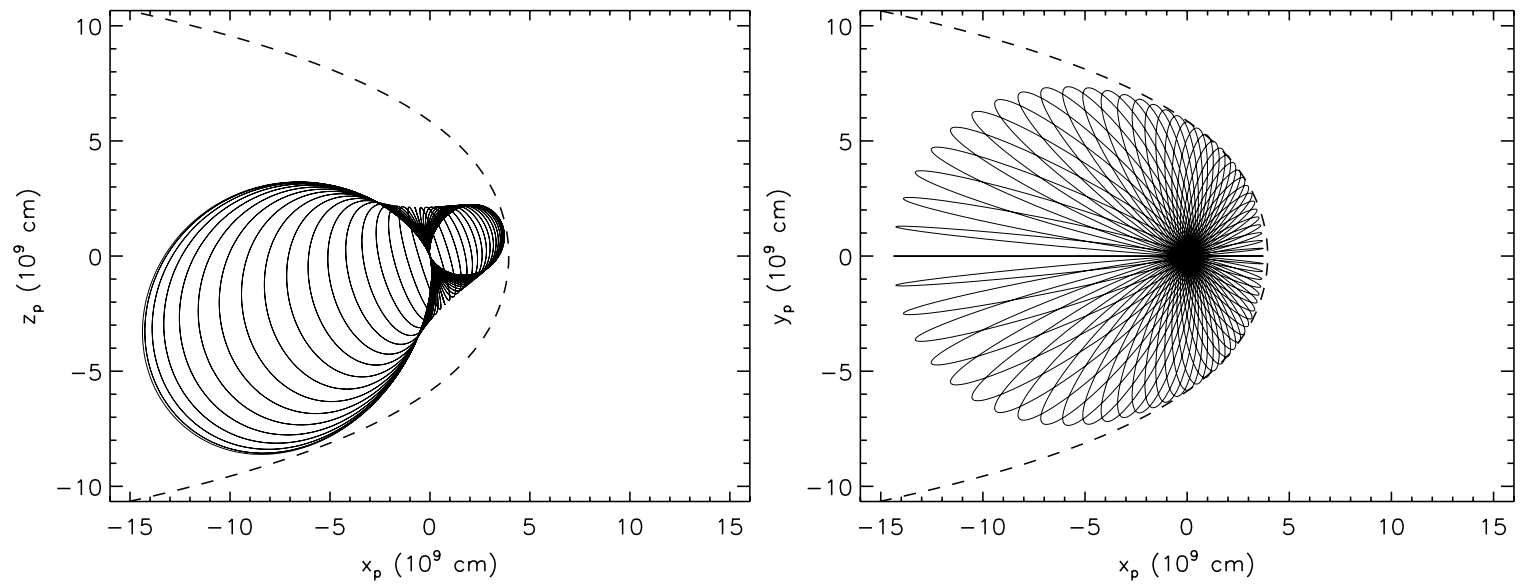

Figure 4. Confined rotating vacuum dipole in the boundary model on MJD 54050 (2006 November 11) - a view in the $x_{\mathrm{p}}-z_{\mathrm{p}}$ plane (left) and a view in the $x_{\mathrm{p}}-y_{\mathrm{p}}$ plane (right). Pulsar B is located at the center of the coordinate system and the wind of A comes toward the $-x_{\mathrm{p}}$ direction. The dashed line shows the derived bow shock from the wind-magnetosphere interaction model and this models the open and closed field lines of the magnetosphere. The solid lines are the last closed field lines with respect to this boundary. The field lines that have higher latitude than these shown closed field lines are considered open field lines. The scale is in units of $10^{9} \mathrm{~cm}$. Here, $\alpha=61^{\circ}$ and $\theta=138.5$; these are our best-fit geometrical parameters from Section 4. This view corresponds to the orbital phase of $223^{\circ}$. Note, for clarity of plots, that we take the spin phase as zero, so that the north pole of the pulsar is pointing below the $z_{\mathrm{p}}=0$ plane. However, the shape of the magnetosphere changes with the spin and orbital motion and over time due to precession.

spin precession, we transform a particular field line to another frame that is fixed with our line of sight. We choose the frame with the $z$-axis parallel to the orbital angular momentum axis and the $x$-axis in the plane of the $z$-axis and the line of sight (see Figure 3(b)). The Cartesian components in this frame are

$$
\begin{aligned}
& x_{\mathrm{o}}=x_{\mathrm{s}} \cos \theta \cos \phi_{\text {prec }}(t)-y_{\mathrm{s}} \sin \phi_{\text {prec }}(t)+z_{\mathrm{s}} \sin \theta \cos \phi_{\text {prec }}(t) \\
& y_{\mathrm{o}}=x_{\mathrm{s}} \cos \theta \sin \phi_{\text {prec }}(t)+y_{\mathrm{s}} \cos \phi_{\text {prec }}(t)+z_{\mathrm{s}} \sin \theta \sin \phi_{\text {prec }}(t) \\
& z_{\mathrm{o}}=z_{\mathrm{s}} \cos \theta-x_{\mathrm{s}} \sin \theta,
\end{aligned}
$$

where $\theta$ is the angle between the spin axis and the orbital angular momentum axis and $\phi_{\text {prec }}(t)$ is the spin precession phase measured from the $x$-axis (i.e., $\phi_{\text {prec }}(t)=0$ when the spin axis is in the plane of $x_{0}-y_{0}$ ). The spin precession phase is given by

$$
\phi_{\text {prec }}(t)=\Omega_{\text {prec }}\left(t-T_{0}\right),
$$

where $\Omega_{\text {prec }}$ is the spin precession rate of $B$, which is 5.061(2) ${ }^{\circ} \mathrm{yr}^{-1}$, and $T_{0}$ is the time when the $\phi_{\text {prec }}(t)$ is zero, defined as the time when the spin axis is in the $x_{0}-y_{0}$ plane. By using the above set of equations, we can transform dipole field lines from the corotating frame of the NS to the orbitfixed frame $\left(x_{0}, y_{0}, z_{0}\right)$ where the $x_{0}-y_{0}$ plane is in the orbital plane and $x_{0} \sin (i)$ is pointing toward the observer, where $i$ is the orbital inclination.

In order to place the polynomial boundary in the $\left(x_{0}, y_{0}, z_{0}\right)$ frame, we need to account for the orbital motion of B. Due to this motion, the orientation of the boundary changes with respect to our line of sight, because the location of A changes with respect to $B$. This relative motion changes the shape of the magnetosphere of B with respect to the line of sight and then the shape of the polar cap region. This results a variation in the emission height estimate across the orbit (more details are given in Section 5). We place the boundary model $\left(x_{\mathrm{p}}, y_{\mathrm{p}}, z_{\mathrm{p}}\right)$ in this orbit-fixed coordinate frame with $z_{\mathrm{o}} \| z_{\mathrm{p}}$ and then rotate it corresponding to the orbital phase $\phi_{\text {orb }}$ with $\left(x_{\mathrm{p}} \cos \phi_{\text {orb }}-y_{\mathrm{p}} \sin \phi_{\text {orb }}, x_{\mathrm{p}} \sin \phi_{\text {orb }}+y_{\mathrm{p}} \sin \phi_{\text {orb }}, z_{\mathrm{p}}\right)$, where $\phi_{\text {orb }}=\psi+90^{\circ}$ (see Figure 3(b)). Here, $\phi_{\text {orb }}$ is measured from the ascending node and $\psi$ is the rotation angle between the $x_{\mathrm{o}}$ and $x_{\mathrm{p}}$ axes. Note that this $90^{\circ}$ angle is included to convert the rotation angle $\psi$ to orbital phase $\phi_{\text {orb }}$ as measured from the ascending node. Then we trace the last closed field lines according to the orientation of the boundary for the given orbital phase. For example, Figure 4 shows the confined magnetosphere in the boundary model with the last closed field lines on MJD 54050 (2006 November 11). Here, we use our best-fit geometry of the pulsar from the beaming model that is described in Section 4. At this particular epoch, the spin precession phase is $\phi_{\text {prec }}=46^{\circ}$ and we use the orbital phase $\phi_{\text {orb }}=223^{\circ}$ and the spin phase $\phi_{\text {spin }}=0$ in the figure. Note that, at this spin and the orbital phases, the spin axis of the pulsar is in the $x_{\mathrm{p}}-z_{\mathrm{p}}$ plane.

The spin of the magnetic axis also results in a change in the shape of the magnetosphere due to the misalignment of the magnetic moment. However, the most important orientation of the magnetic axis is when it reaches the closest approach to our line of sight (i.e., where we detect the emission). In order to measure the point of this closest approach, or the impact parameter $\beta(t)$, we use the equations

$$
\begin{aligned}
& \cos \zeta(t)=\sin \theta \cos \phi_{\mathrm{prec}}(t) \sin i+\cos \theta \cos i \\
& \beta(t)=\zeta(t)-\alpha
\end{aligned}
$$

Here, $\zeta(t)$ is the angle between the spin axis and our line of sight at a given time and the other angles have the usual meaning. We calculate the spin phase that gives this particular closest approach, so that we can estimate the emission height only at this particular spin phase.

Now we can transform field lines from the corotating frame of the NS to the orbit-fixed frame. By using the boundary model, we can trace the last closed field lines, which determine the shape of the magnetosphere. Due to different orientations of the magnetic axis with spin, the shape of the magnetosphere with respect to our line of sight changes, but we are interested only in the spin phase that gives the closest approach to us. Nevertheless, orbital motion and spin precession change the shape and we need to account for these in emission height calculation.

\section{RE-ANALYSIS OF THE BEAM SHAPE}

For the emission height calculation, we require the angular radius of the emission beam. Perera et al. (2010) claimed the beam shape of B to be elliptical by modifying the Clifton \& 


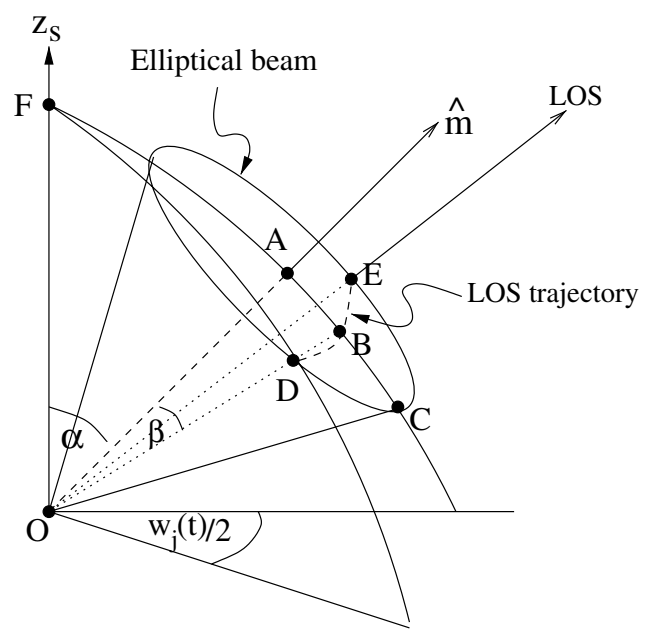

Figure 5. Elliptical emission cone in the frame $\left(x_{\mathrm{s}}, y_{\mathrm{s}}, z_{\mathrm{s}}\right)$. The magnetic axis of the beam represents with $\hat{m}$ and it is misaligned with the spin axis of an angle $\alpha$. The trajectory of the line of sight across the beam due to rotation is denoted with $D B E$. The angle $B \hat{O} D$ is the longitudinal angular radius $\rho_{\mathrm{l}, j}(t)$ of the beam for a given intensity level at a given time. The angle $A \hat{O} C$ is the angular radius across the semimajor axis of the beam $\rho_{\mathrm{a}, j}$ for a given intensity level, which is time independent and fixed for the beam. The angle $A \hat{O} D$ is the effective angular radius of the beam $\rho_{\mathrm{e}, j}(t)$ for a given intensity level.

Weisberg (2008) geometrical framework. The model in Perera et al. (2010) used the two-dimensional geometry of the beam after projecting it to a plane which is perpendicular to the spin axis, resulting in a projected angular radius. We improve this model by using a more realistic three-dimensional model in this paper in order to determine the actual angular radius.

The angular radius of a circular emission beam has been calculated using pulse profile widths and an assumed emission geometry (Gil et al. 1984). Since Perera et al. (2010) showed that the shape of B's beam is not circular, we attempt to derive an approximate equation for an elliptical beam in threedimensional and then follow the same analysis of Perera et al. (2010) in order to determine the geometry. First, we construct the beam with a set of coaxial hollow cones representing different intensity levels in a way such that the outermost one represents the lowest intensity level and then the intensity increases gradually inward toward the maximum and then decreases until reaching the center of the beam. All these cones have cross sections with a constant ellipticity of $a_{j} / b_{j}$, where $a_{j}$ and $b_{j}$ are semimajor and semiminor axes, respectively, of each hollowcone beam. Then, for any given longitudinal angular radius $\rho_{\mathrm{l}, j}(t)$ (see Figure 5), magnetic misalignment angle $\alpha$, and impact parameter $\beta(t)$, the pulse profile width $w_{j}(t)$ can be derived from spherical trigonometry (i.e., from spherical triangle FBD in Figure 5) as follows:

$$
w_{j}(t)=2 \arccos \left(\frac{\cos \left(\rho_{1, j}(t)\right)-\cos ^{2}(\alpha+\beta(t))}{\sin ^{2}(\alpha+\beta(t))}\right),
$$

where subscript $j$ specifies different intensity levels of the pulse profile. Note that $\rho_{\mathrm{l}, j}(t)$ is time dependent because the region where our line of sight cuts the beam is changing with time due to precession. In order to relate $\rho_{\mathrm{l}, j}(t)$ with the elliptical beam shape, we derive an equation with the assumption that the crosssection of the beam is small enough to use one-dimensional trigonometry. Then the longitudinal angular radius $\rho_{\mathrm{l}, j}(t)$ can be given as a function of $\beta(t)$,

$$
\rho_{\mathrm{l}, j}(t)=\frac{1}{\chi} \sqrt{\sin ^{2}\left(\rho_{\mathrm{a}, j}\right)-\cos ^{2}\left(\rho_{\mathrm{a}, j}\right) \tan ^{2}(\beta(t))},
$$

where $\rho_{\mathrm{a}, j}$ is the angular radius across the semimajor axis of the beam (see Figure 5) for a given intensity level and $\chi=a_{j} / b_{j}$, which is a constant for all different intensity cones. This expression shows that the minimum $\rho_{\mathrm{l}, j}(t)$ of zero occurs when the line of sight just encounters the beam (i.e., $\left.\beta(t)=\rho_{\mathrm{a}, j}\right)$, resulting in $w_{j}(t)=0$. The maximum $\rho_{\mathrm{l}, j}(t)$ occurs when the line of sight crosses the center of the beam (i.e., $\beta(t)=0$ ), which leads to the maximum $w_{j}(t)$. Therefore, by combining Equations (11) and (12) for a given $\alpha, \beta(t)$, and $\rho_{\mathrm{a}, j}$, we can calculate the pulse profile width $w_{j}(t)$ for any given intensity level.

In order to determine the geometry of B, we fit the modelpredicted pulse profile widths to observed pulse profile widths of BP1 at different intensity levels using the same likelihood analysis that was described in Perera et al. (2010). The fit was done by searching the entire parameter space of $\alpha, \theta, \chi$, and $T_{0}$. For each combination of these parameters, we vary $\rho_{\mathrm{a}, j}$ from $0^{\circ}$ to $30^{\circ}$ freely until we reach the best solution. Then we use a maximum likelihood analysis to determine the best-fit geometrical parameters. The best-fit model for BP1 is shown in Figure 6. The estimated geometrical parameters $\alpha=61.0_{-2.4}^{+7^{\circ} .9}$ and $\theta=138.5_{-4.4}^{+5^{\circ} .3}$ are consistent with those in Perera et al. (2010) and Breton et al. (2008) within the $2 \sigma$ errors. The ratio $\chi$ is constrained to be $2.6_{-0.6}^{+0.4}$, lower than the estimate of the previous paper. Our new estimate is more believable because it has been derived from a full threedimensional viewing model. In addition to these parameters, we derive $T_{0}$ to be MJD $57399_{-25}^{+4}$ (2016 January 12), which results in a precessional phase of $46^{\circ}$ at an epoch of MJD 54050 (2006 November 11). This estimate is consistent with the value predicted by Breton et al. (2008) at the same epoch. However, this is a somewhat arbitrary parameter that can be chosen from our best-fit model. Note that the best-fit $T_{0}$ in Perera et al. (2010) is about MJD 33360 (1950 March 20), which results in a precessional phase of $73^{\circ}$ at an epoch of MJD 54050. These two best-fit $\phi_{\text {prec }}$ result in a shift of the hour-glass two-dimensional pulse profile shape along the precessional phase or time axis (see Figure 6 of this analysis and Figure 17 of Perera et al. 2010).

The angular radius across the semimajor axis of the beam at the maximum intensity level $\rho_{\mathrm{a}, 100}$ and $10 \%$ of the maximum $\rho_{\mathrm{a}, 10}$ are constrained to be 9.9 and 14.3 , respectively. In order to determine the effective angular radius of the beam (more details are given in Section 5), we use these angular radii with the derived beam geometry.

For emission height estimates for normal non-precessing pulsars, a beam shape is not essential because our line of sight always observes the same section of the emission beam. However, for precessing pulsars, we must consider a beam shape in order to determine the emission height due to observing different sections of the emission beam. Therefore, in this particular case, we use our derived elliptical beam shape with the best-fit geometrical parameters of B to estimate emission heights in Section 5. As we see in Perera et al. (2010), the pulse profile evolution is somewhat similar in both bright phases. Therefore, we use the above best-fit beam parameters from BP1 in our emission height estimates for both bright phases.

Perera et al. (2010) reported that the radio emission of $\mathrm{B}$ disappeared in 2008 March, because the line of sight precessed 


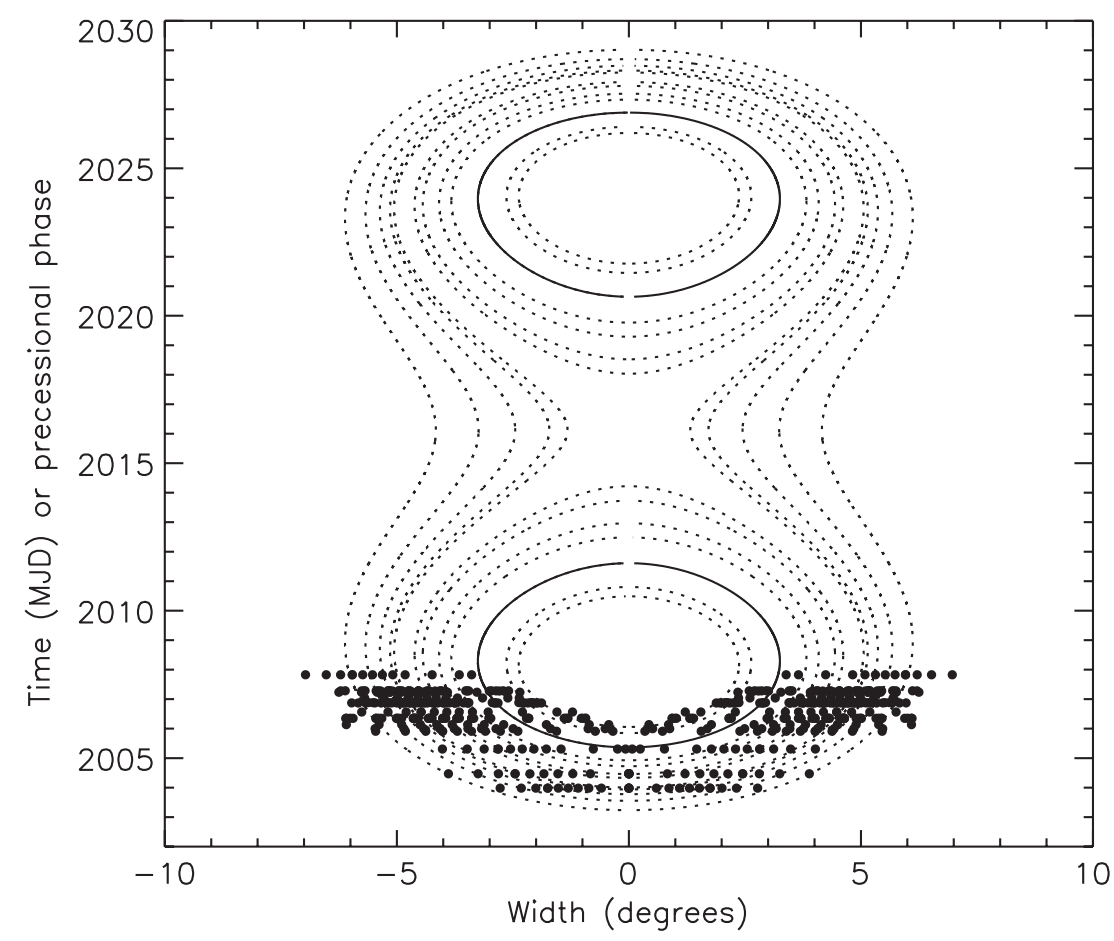

Figure 6. Two-dimensional pulse profile of the orbital longitude region $185^{\circ}-235^{\circ}$ (BP1), assuming the elliptical hollow-cone beam. The best-fit geometrical parameters are $\alpha=61.0_{-2^{\circ} .4}^{+7^{\circ} .9}, \theta=138.5_{-4^{\circ} .4}^{+5^{\circ}}$, and $\chi=2.6_{-0.6}^{+0.4}$ (errors are $1 \sigma$ ). The corresponding $T_{0}$ is MJD 57399 (2006 November 11), which is the time where the spin axis of the pulsar is in the plane of our line of sight and the orbital angular momentum axis. Note that these $\alpha$ and $\theta$ values are consistent with previous results (Perera et al. 2010; Breton et al. 2008). The dots are the widths at equal intensity levels of the observed pulse profile. Each horizontal row of dots represents an observation at a given epoch. Equal-intensity contours are the elliptical beam model-predicted pulse profile widths at different intensities. The intensity increases from the inner dashed line outward until the first solid line, which is the intensity of the peak, and then decreases outward again. The intensity levels are, from the inner dashed line, $80 \%, 90 \%, 100 \%, 90 \%, 80 \%, 70 \%, 60 \%, 50 \%, 40 \%, 30 \%, 20 \%$, and $10 \%$. The vertical axis is calibrated in years and can also be considered the spin precession phase. Note that this figure shows the emission from the full elliptical beam. If the beam is partially filled according to 2008 radio disappearance, then the model predicts no radio pulse profiles from 2008 to 2024 .

away from the partially radio-filled elliptical beam. According to the same argument that the beam is not entirely radio loud, we can explain the radio disappearance with our new elliptical beam geometry. With the partially filled beam configuration, we can predict the reappearance of the radio emission as our line of sight precess back to the radio loud region of the beam. With the model described in Perera et al. (2010), the reappearance is predicted to occur in around 2035 with the same part of the beam or in around 2014 if the beam has two symmetric radio-filled portions. However, our new model describes that the reappearance is predicted to occur in around 2024 with the same part of the beam. Therefore, Figure 6 changes with the partially filled horse-shoe beam to non-detectable emission from 2008 to 2024. If our new beam model is correct, then the beam should not have two symmetric radio-filled parts, because our line of sight crossed around the center of the beam when the disappearance occurred in 2008. Therefore, if there are two symmetric parts, then we would be able to detect radio emission at present day. The two different predictions for the reappearance from the two models mainly occur due to two different best-fit $T_{0}$ values. These two different $T_{0}$ values give two different solutions for the geometry of $\mathrm{B}$, however, the best-fit values for parameters $\alpha$ and $\theta$ are the same for the two models with the $2 \sigma$ uncertainty.

\section{EMISSION HEIGHT CALCULATION}

In order to estimate the radio emission heights of pulsar B, we use the previously defined boundary model, the field line tracing technique, and the modeled geometry of the beam. As we mentioned earlier, we assume that the radio emission is produced above the polar cap region and originates tangential to the local magnetic field lines.

First, we need to determine the boundary of the polar cap region, given by the last closed field lines. This can be done by tracing the field lines with the derived boundary model as described in Section 3.1. We assume that the radio emission comes from above the entire polar cap region, so that the outer edge of the pulse profile (i.e., $10 \%$ of the maximum intensity) corresponds to the region between the open and closed field lines, approximately equal to the last closed field line. Then we determine the emission height that originates from these last closed field lines.

In order to determine the tangent to a particular last closed field line at a given moment, we rewrite the coordinate transformations in Section 3.1 in matrix form (see, e.g., Gangadhara 2004), so that it is easy to evaluate the equations relevant for our calculation. First, we write the dipole field equation in the corotating frame of the NS as

$$
\overrightarrow{r_{\text {cor }}}=r_{0}\left(\sin ^{3} \lambda \cos \phi, \sin ^{3} \lambda \sin \phi, \sin ^{2} \lambda \cos \lambda\right) .
$$

We then transform it to the orbit-fixed frame

$$
\overrightarrow{r_{\mathrm{orb}}}=A \cdot B \cdot \overrightarrow{\mathrm{cor}}
$$

where

$$
A=\left(\begin{array}{ccc}
\cos \alpha \cos \phi_{\text {spin }} & -\sin \phi_{\text {spin }} & \sin \alpha \cos \phi_{\text {spin }} \\
\cos \alpha \sin \phi_{\text {spin }} & \cos \phi_{\text {spin }} & \sin \alpha \sin \phi_{\text {spin }} \\
-\sin \alpha & 0 & \cos \alpha
\end{array}\right)
$$


and

$$
B=\left(\begin{array}{ccc}
\cos \theta \cos \phi_{\text {prec }} & -\sin \phi_{\text {prec }} & \sin \theta \cos \phi_{\text {prec }} \\
\cos \theta \sin \phi_{\text {prec }} & \cos \phi_{\text {prec }} & \sin \theta \sin \phi_{\text {prec }} \\
-\sin \theta & 0 & \cos \theta
\end{array}\right) .
$$

For the detection of radio emission, our line of sight must be parallel to the tangential vector of the given field line at a particular point. By locating this point on the field line, we can determine the height of the radio emission. To evaluate the tangent to the field line, we take $\overrightarrow{r_{t}}=\partial \overrightarrow{r_{\text {orb }}} / \partial \lambda$. Then the unit vector along the tangential direction $\left(\hat{r_{t}}=\overrightarrow{r_{t}} /\left|\overrightarrow{r_{t}}\right|\right)$ can be written as

$$
\hat{r}_{t}=A \cdot B \cdot \sqrt{\frac{2}{5+3 \cos (2 \lambda)}}\left(\begin{array}{c}
3 \cos \phi \sin \lambda \cos \lambda \\
3 \sin \phi \sin \lambda \cos \lambda \\
2 \cos ^{2} \lambda-\sin ^{2} \lambda
\end{array}\right) .
$$

The direction of the magnetic moment axis in the orbit-fixed frame can be written as

$$
\hat{m}=A \cdot B \cdot \hat{z}_{\mathrm{o}} .
$$

Then we evaluate the angle between the direction of the magnetic moment axis and the vector tangential to the field line $(\tau)$ at any time through the expression

$$
\cos (\tau)=\hat{r}_{t} \cdot \hat{m}=\frac{1+3 \cos (2 \lambda)}{\sqrt{10+6 \cos (2 \lambda)}} .
$$

This is the same as Equation (8) in Gangadhara (2004). At the point of detection of radio emission, we take the angle $\tau$ to be equal to the effective angular radius of the previously derived emission beam at the given time. According to our assumption that the outer edge of the pulse profile ( $10 \%$ maximum) comes from the last closed field line, we take $\tau \approx \rho_{\mathrm{e}, 10}(t)$, where $\rho_{\mathrm{e}, 10}(t)$ is the effective angular radius of the beam (angle $A \hat{O} D$ of Figure 5) at the $10 \%$ of the maximum intensity level corresponding to a particular impact parameter $\beta(t)$. We can derive an equation for $\rho_{\mathrm{e}, 10}(t)$ by using the spherical triangle $F A D$ of Figure 5 as

$$
\begin{aligned}
\cos \left(\rho_{\mathrm{e}, 10}(t)\right)= & \cos (\alpha) \cos (\alpha+\beta(t)) \\
& +\sin (\alpha) \sin (\alpha+\beta(t)) \cos \left(w_{10}(t) / 2\right) .
\end{aligned}
$$

Here, $w_{10}(t)$, the model-estimated pulse width, can be found through Equations (11) and (12) with the best-fit parameters $\alpha, \theta, \chi$, and $\rho_{\mathrm{a}, 10}$. The impact parameter $\beta(t)$ for the given time can be determined through Equations (9) and (10) with the best-fit $T_{0}$. This $w_{10}(t)$ is simply the $10 \%$ pulse width of the two-dimensional pulse profile given in Figure 6 at the given time. By simplifying Equation (19), we find an expression for $\lambda$, which is the colatitude of the emission point. This expression can be written as

$$
\cos (2 \lambda)=\frac{1}{3}\left[\cos \left(\rho_{\mathrm{e}, 10}(t)\right) \sqrt{8+\cos ^{2}\left(\rho_{\mathrm{e}, 10}(t)\right)}-\sin ^{2}\left(\rho_{\mathrm{e}, 10}(t)\right)\right] .
$$

This is same as Equation (9) in Gangadhara (2004), so that the colatitude angle of the emission point in our complicated geometry is simplified to that of an isolated pulsar. Then the emission height of this point can be calculated by using the first equation of (5). However, determining the field line constant, $r_{0}$, in this equation is difficult due to the bow shock boundary and its variation. Kijak \& Gil (1997) assumed that this $r_{0}$ is the light cylinder radius since the isolated pulsars that they have studied have low magnetic inclinations. To determine $r_{0}$ for our particular case, we trace the last closed field line that is tangent to our line of sight at the closest approach of the magnetic moment with respect to the bow shock boundary. Then we use the dipole field equation to estimate the emission height.

The orientation of the bow shock changes across the orbit with respect to our line of sight at the closest approach, so that the emission height changes with orbital longitude, because the last closed field lines are defined with respect to the bow shock. Moreover, when the central part of the radio beam crosses our line of sight, we will detect a double-peaked profile since the two edges, leading and trailing, of the beam cross our line of sight. Thus due to the different orientation of the magnetic moment axis at these two edges with respect to us, our line of sight is tangent to two different last closed field lines which have two different $r_{0}$ values. Thus, the height of the emission produced by the leading and trailing edges of the beam are different. This is shown in Figure 7. For example, the emission heights produced by the leading components of the beam are constrained to be in a range of $[24 \pm 8,31 \pm 10]$ and $[20 \pm 6,21 \pm 7]$ in NS radii $(10 \mathrm{~km})$ on MJD 54050 (2006 November 11) for BP1 and BP2, respectively. The heights of the trailing edge of the beam in BP1 and $\mathrm{BP} 2$ are constrained to be in a range of $[15 \pm 5,19 \pm 6]$ and $[21 \pm 7,38 \pm 12]$ in NS radii, respectively. The errors of the height estimates are calculated from the $1 \sigma$ uncertainties of the best-fit geometrical parameters from the beaming model. Thus, the uncertainty of the height estimate is in a range of $[6,10]$ and $[5,19]$ in NS radii for the leading and the trailing edge of the beam, respectively, across the orbit on this particular day.

Also, due to precession of the spin axis, the emission height varies with time because the angle $\beta(t)$ varies with time. Again, there are two different heights for the leading and trailing edges of the beam. These are shown in Figures 8 and 9. In BP2, the difference between the two heights is not as significant as in $\mathrm{BP} 1$ because of the orbital position of B in BP2. In this region, pulsars B, A, and our line of sight are roughly aligned, resulting a less deviation in emission heights for the two edges of the beam.

If the emission is produced from the boundary between the open and closed field lines, we can consider these estimates to be the actual emission heights for B. If the emission is produced elsewhere within the open field line region, these are lower limits on emission heights.

\section{UPPER LIMIT OF THE EMISSION HEIGHT}

We may also set an upper limit to the radio emission height by modeling the distortions of B's magnetosphere induced by the wind of A. Since both the magnetosphere and the wind are strongly magnetized, the distortions depend on the relative strengths of the magnetic fields and thus on the distance from the NS. Depending on the location of the radio emission region and the line of sight (and hence on the orbital position) an observer will detect different radiation signatures of the distorted magnetosphere. Inversely, by studying the orbital modulation and using a model of the distorted magnetosphere, we can deduce the location of the emission region.

Similar to how the Sun distorts Earth's magnetosphere, pulsar A produces a strong enough wind to interact with the magnetic field of $\mathrm{B}$ and shape its magnetosphere. The nature of this interaction will vary depending on the properties of the wind. For a wind with a substantial particle flux, the formation of a bow shock, similar to case of Earth, is expected. In this 


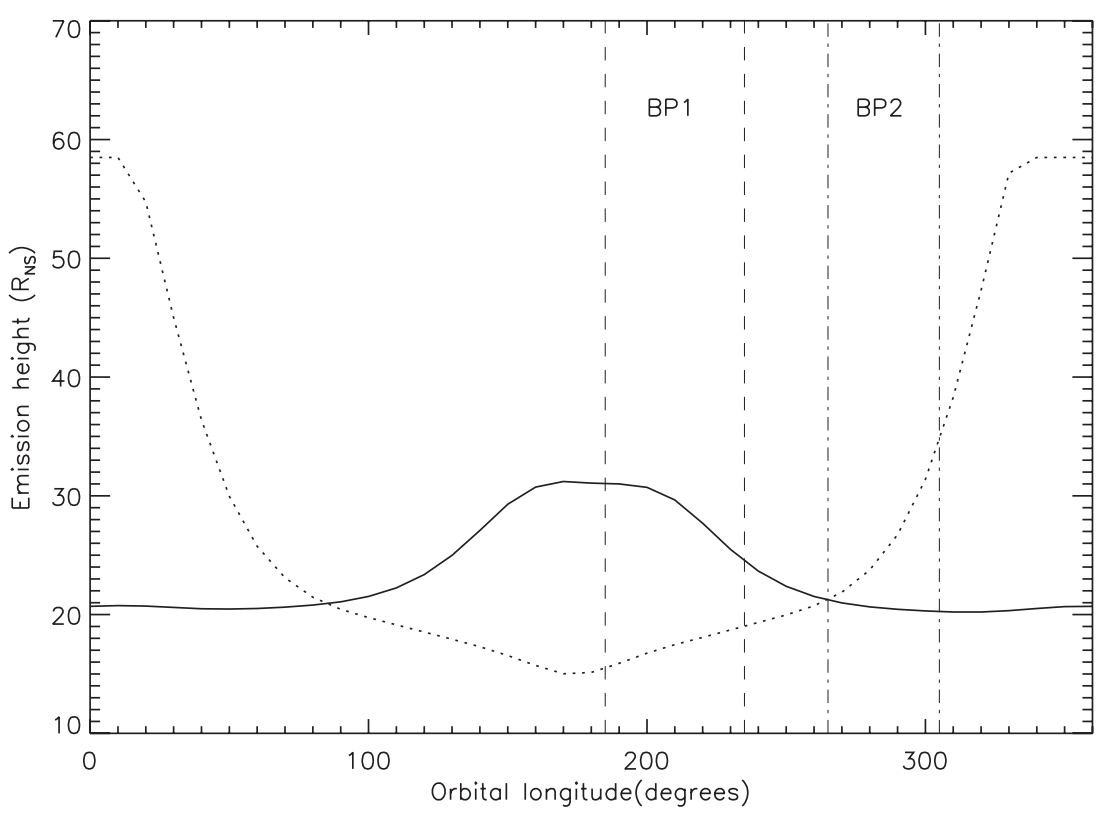

Figure 7. Model-estimated radio emission height across the orbit on MJD 54050 (2006 November 11). The solid line represents the height of the emission produced by the leading edge of the beam and the dotted line represents that for the trailing edge of the beam. In order to consistent with observations, we used our best-fit geometrical parameters of the beaming model, $\alpha=61^{\circ}$ and $\theta=138.5$. At this epoch, $\phi_{\text {prec }}$ is $46^{\circ}$, the impact parameter $\beta$ is $3^{\circ}$, and the corresponding spin phase is $126^{\circ}$. Note that the difference between the corresponding heights for the leading and trailing components of the beam is more significant in some parts of the orbit. The orbital longitude regions for BP1 and BP2 are denoted with dashed and dot-dashed lines, respectively.

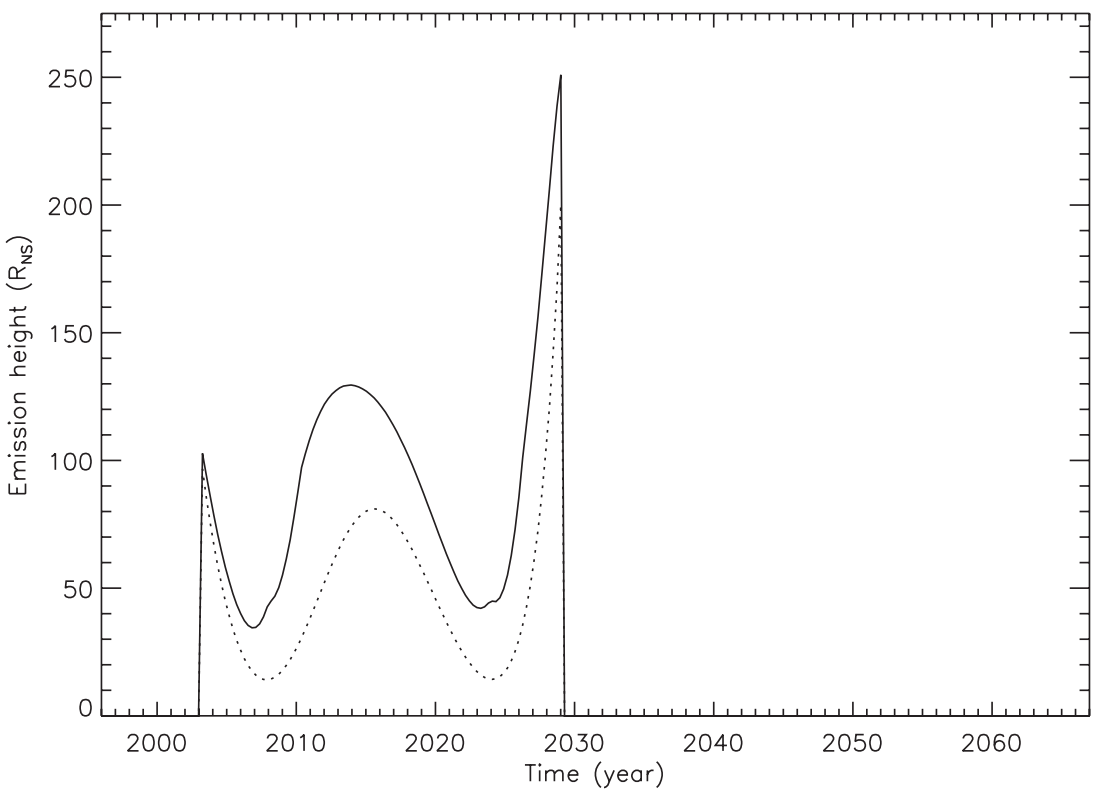

Figure 8. Model-estimated radio emission heights of the orbital longitude region of BP1 vs. time across one precessional cycle. This predicted variation of emission height with time is due to spin precession, making different line-of-sight cuts across the radio beam. The solid line represents the emission height from the leading edge of the beam and the dotted line represents that for the trailing edge. Here, the orbital phase is fixed at $200^{\circ}$ (BP1), but the spin phase changes with time correspond to the $\beta$ value. The time axis represents a full precession cycle, 71 yr. Here, we have used the same geometrical parameters as in Figure 7 and assumed a full elliptical beam, not a partially filled horse-shoe beam. If the beam is partially filled then no radio emission is expected from 2008 to 2024 . This is why the model still predicts radio emission at present-day MJDs. The emission height is zero ( 2030-2067) when the line of sight is out of the radio beam.

MHD confinement model, the shape of Earth's magnetosphere is mostly determined by the pressure balance between the supersonic solar wind and Earth's nearly dipolar field. This curved shape of the magnetosphere is reproduced well by current numerical models (Tsyganenko 2002a, 2002b; Tsyganenko \& Sitnov 2007).

On the other hand, for a strongly magnetized wind, reconnection between the wind and the companion's magnetic field lines must be considered. This results in an open structure for the whole magnetosphere, similar to the one originally proposed by Dungey (1961) for planetary magnetospheres.

In the case of the double pulsar, it is unclear whether an MHD confinement model or a reconnection model is more applicable due to the unknown composition of A's wind. However, we are mostly interested in the overall geometric structure of B's magnetosphere. For this purpose, it is sufficient to discuss magnetospheric structure in the most basic terms, relying on the models of planetary magnetospheres. We consider two extreme, though 


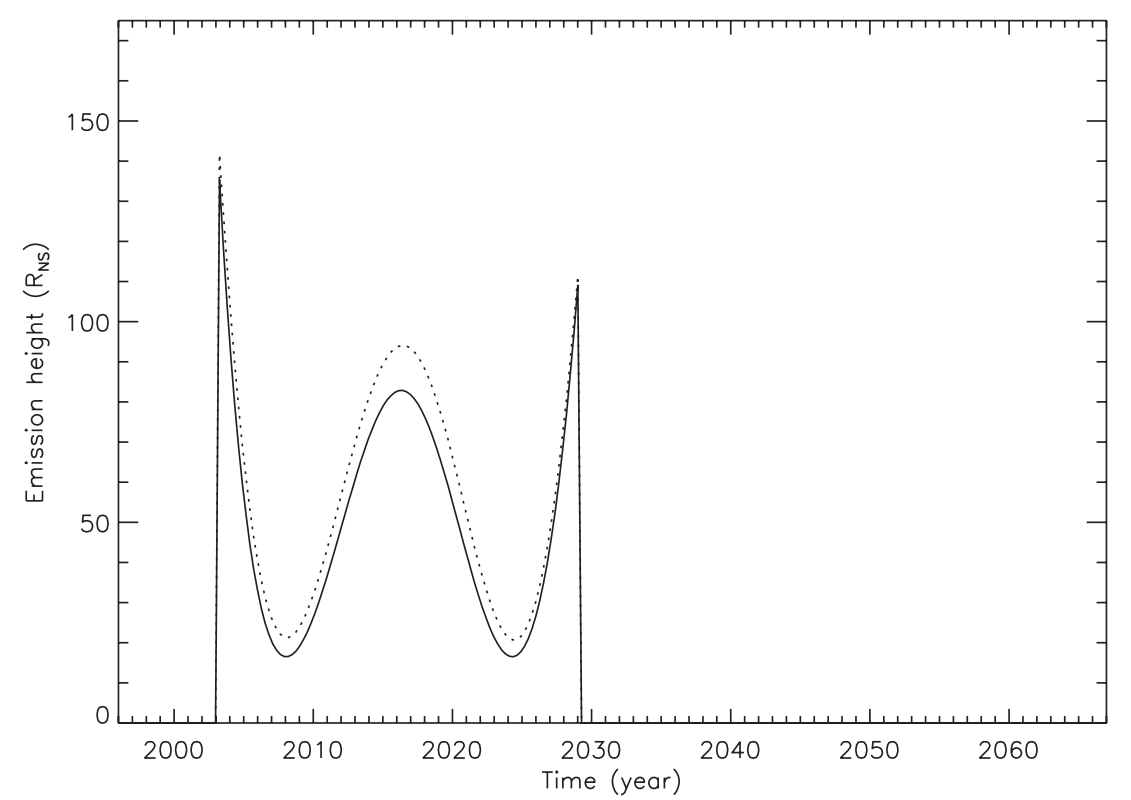

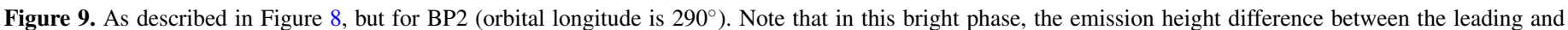
trailing components of the beam is not as significant as in BP1.

complimentary, models of Earth's magnetosphere; the highly resistive, analytical reconnection model of Dungey (1961), hereafter D61, and the fully screened, three-dimensional numerical hydrodynamic confinement model of Tsyganenko (2002a, 2002b), hereafter TS02.

We use the D61 model of the open planetary magnetosphere as a simple analytical representation of the distorted magnetosphere. The D61 model states that the interplanetary magnetic field (IMF) may become reconnected with the terrestrial field along the dayside magnetopause, where the magnetopause is the boundary between Earth's magnetosphere and the solar wind. This results in a distortion of the higher altitude regions of the inner magnetosphere.

Forbes \& Speiser (1971) neglected the dynamics of the reconnection processes and modeled Earth's magnetosphere as a linear superposition of two magnetic fields: Earth's closed field and the solar wind's uniform field. Following this approach, we can represent B's magnetosphere as a simple addition of the pulsar's dipole field and the wind's uniform field. Similar to the IMF in the D61 model, we treat the magnetic field in A's wind as homogeneous in the vicinity of $\mathrm{B}$, with the direction of the magnetic flux density vector perpendicular to the line connecting the two pulsars (assuming a toroidal field). However, depending on whether the large-scale toroidal field is prograde or retrograde with respect to the orbit, the geometric structure of the magnetosphere can be significantly different (see Figure 10).

Alternatively, we can use TS02 for more precise, threedimensional modeling. This model is a data-based best-fit representation for Earth's screened magnetosphere based on a large number of satellite observations. The model provides the option of adding the contributions from external magnetospheric sources such as the ring current, magnetotail current system, magnetopause currents, and the large-scale system of fieldaligned currents to Earth's dipole field.

We used the GEOPACK code repository developed by Tsyganenko, with modifications to match the properties of the double pulsar system. Instead of analyzing every current component in the TS02 model separately, we manipulated the global input parameters of the code that define the geometric structure of the magnetosphere. The shape and scale of the magnetosphere is controlled by the solar wind ram pressure and the dipole tilt only. Variations in the value of the ram pressure change the magnetosphere self-similarly. In the numerical model, the ram pressure is represented by the parameter PARMOD(1) and has units in $\mathrm{nPa}$. PARMOD(2) represents the disturbance storm time (Dst) index and has units in nT. The Dst index is a measure of the size and strength of the ring current, which contributes to the overall field configuration in the inner magnetosphere. The TS02 model is designed in such a way that the structure of the magnetosphere within a stand-off distance from the Earth has a very small dependence on the components of the IMF. Hence, for simplicity we set the transverse components of the external field $\left(\operatorname{PARMOD}(3)=B_{y}\right.$ and $\left.\operatorname{PARMOD}(4)=B_{z}\right)$ to zero.

We performed a visual fitting (see Figure 11) of the boundary produced by the TSO2 code to the boundary produced by our theoretical model (Equations (1) and (2) in Section 3). We set PARMOD(3) and PARMOD(4) equal to zero and changed PARMOD(1) and PARMOD(2) until the shapes of the boundaries matched. The best-fit values that we obtained are PARMOD $(1)=8 \mathrm{nPa}$ for the solar wind ram pressure, $\operatorname{PARMOD}(2)=100 \mathrm{nT}$ for the Dst index, and, by default, the zero transverse components of the IMF $(\operatorname{PARMOD}(3)=$ $0 \mathrm{nT}$, PARMOD $(4)=0 \mathrm{nT})$. This set of parameters produces a magnetosphere boundary with a stand-off distance of about 10.4 stellar radii. In order to make the spatial scaling consistent with the properties of the double pulsar, we rescaled the stellar radius parameter $R 0$ from 1 to 0.0026 . This change simply ensures that the stand-off distance is about 4000 stellar radii, which is the value assumed throughout this section.

The obtained values of the parameters (PARMOD(1-4) and $R 0)$ are not supposed to be physically realistic; rather, they produce a magnetosphere with a shape and size (defined by the stand-off distance) that match the properties of the double pulsar. Moreover, there could be other successful fits since they are derived from the visual inspection of the boundaries (see Figure 11). Nevertheless, using this particular set suits our purpose of modeling an approximate structure of pulsar 


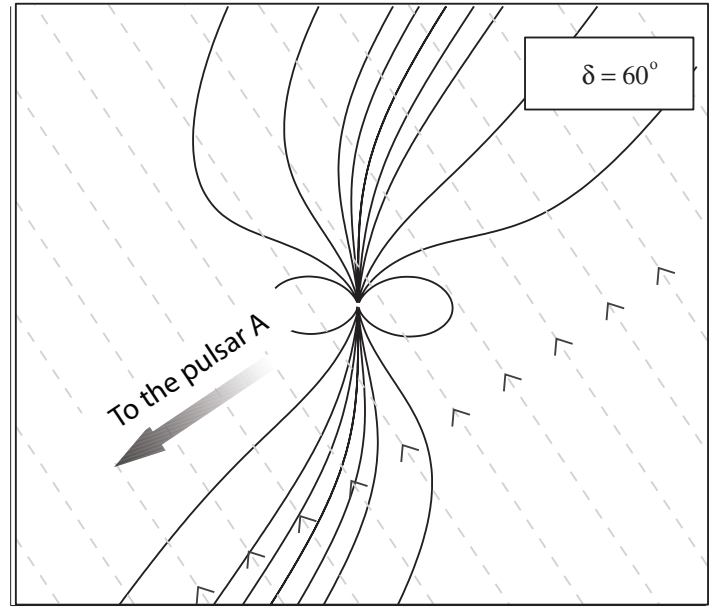

(a)

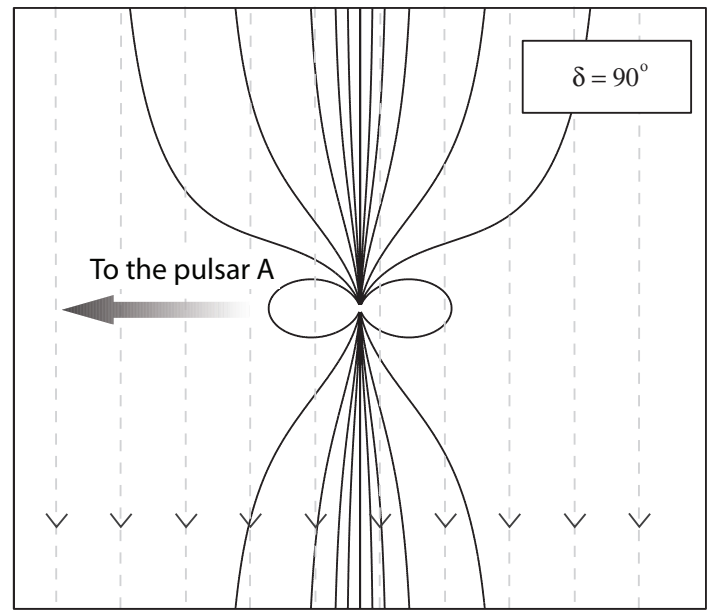

(c)

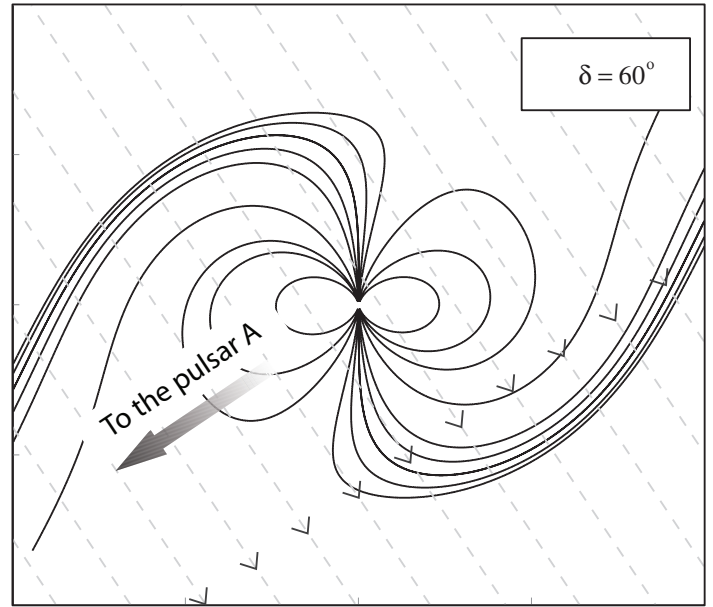

(b)

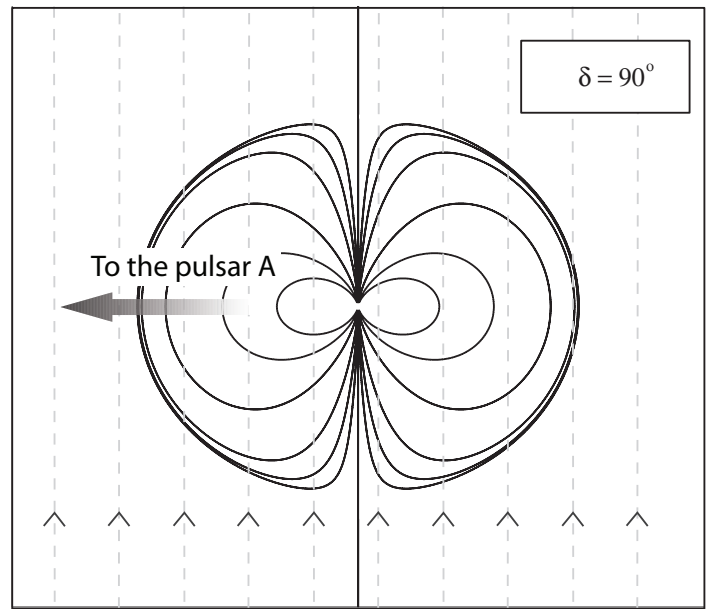

(d)

Figure 10. Geometric structures of Dungey-type magnetospheres in two dimension. Magnetospheric models constructed by adding a wind's uniform field, in the direction shown by the arrows on the dashed lines, to a dipole field with northward orientation. Magnetic fields of $6.4 \times 10^{11} \mathrm{G}$ and $10 \mathrm{G}$ are assumed for the surface magnetic field of pulsar B and the wind magnetic field, respectively. The direction of the wind is the opposite of the arrow showing the direction to the pulsar A. Both (a) and (b) have the same direction of the wind and orientation of the dipole. Same is true for (c) and (d). However, two different possible orientations of the magnetic field in the wind result in very different overall magnetospheric structures. Panel (a) shows smaller deflection of the polar field lines compared to (b). Magnetosphere in (c) is mostly open, whereas one in (d) is mostly closed. In the latter, a radius of the enclosed magnetosphere is about $4 \times 10^{9} \mathrm{~cm}$.

B's distorted magnetosphere without using large computational resources.

We employed the same criteria to estimate an upper limit for the emission height for both models. We assumed that the elliptical emission beam is located close to the polar field lines, which are nearly aligned with the magnetic axis at $r \ll R_{\mathrm{LC}}$, where $R_{\mathrm{LC}}$ is the light cylinder radius. The anisotropic distortion of the magnetosphere by the wind changes the location of the polar field line relative to the undistorted magnetic axis (see Figures 12(b) and (d)). As a first approximation, the deflection angle can be expressed as $\alpha_{\mathrm{defl}} \sim B_{w} / B_{\mathrm{p}}$, where $B_{w}$ and $B_{\mathrm{p}}$ are the magnetic filed of pulsar A's wind and the magnetic field of pulsar B, respectively. Close to the NS's surface, the influence of the wind's magnetic field on the overall field structure is negligible. Therefore, $\alpha_{\text {defl }} \sim 0$ at the surface and increases outward as the wind's magnetic field becomes comparable to the pulsar field near the boundary. The ratio between the two fields, and hence the amplitude of the deflection, depends on the distance from the star as well as on $\delta$, the angle between B's magnetic axis and the line connecting the two pulsars. There is a certain height above which the distortion is strong enough to deflect the polar field line by more than the angular radius of the beam $\left(\rho_{\mathrm{a}, 10} \simeq 14.3\right)$, which is determined in Section 4 . Furthermore, if the component of the distortion perpendicular to the trajectory of the center of the beam in the vicinity of our line of sight is large enough, then the emission beam can be pushed away from the line of sight to the extent that they do not intersect with each other for any spin phase (see Figure 12(b)). This will render the emission beam unseen. On the other hand, the opposite can be true if the distortion occurs mostly along the local trajectory of the beam. In this case, the visibility of the beam stays unchanged and a small shift in the spin phase, at which the emission beam is seen, might be the only observable imprint of the distortions (Figure 12(d)). However, the geometry of pulsar B suggests that the former must be realized (Figures 12(a) and (b)). Therefore, in order to be able to detect pulsed radio emission from $\mathrm{B}$, the deflection angle $\alpha_{\text {defl }}$ should not exceed 14.3 . This places an upper limit on the height of the emission region. However, in order to be able to use this reasoning, a pulsar must be detectable through its pulsed radio emission. We therefore restrict our analysis to only BP1 and BP2, the distinct radio-loud regions of the orbit. 


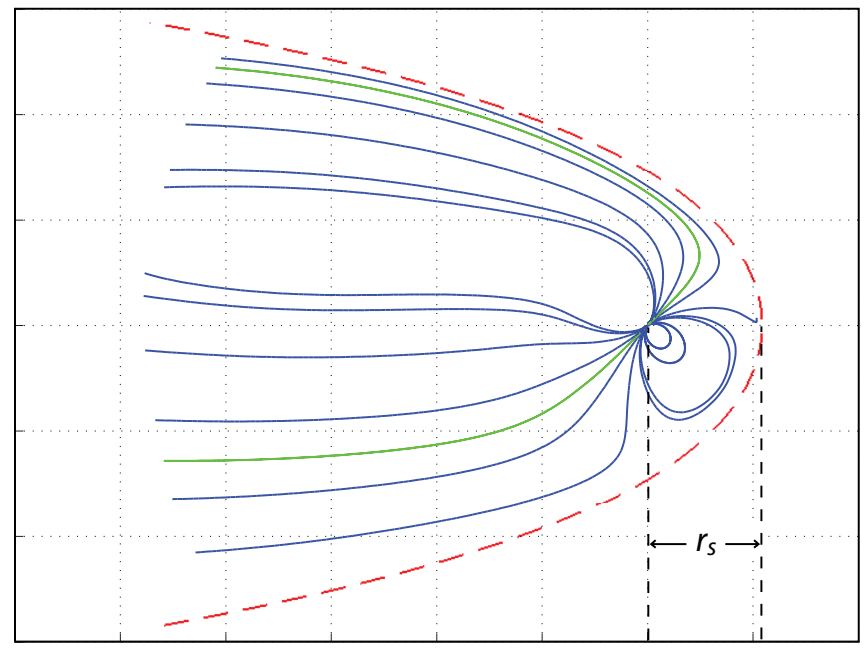

Figure 11. TS02 magnetosphere fitted to the theoretical boundary model. Field lines are plotted in solid. Out of which the green color is for the polar field lines. Dashed line represents the bow shock boundary model. Tilt of the dipole is $45^{\circ}$ and $r_{\mathrm{s}} \sim 4 \times 10^{9} \mathrm{~cm}$. We fix the shape and scale of the boundary and fit the parameters of the TS02 model.

(A color version of this figure is available in the online journal.)
In order to make use of this criteria, we analyzed the moments of the closest approach at the orbital phases within BP1 and BP2. At the moment of the closest approach, the magnetic axis is nearly aligned with the line of sight, making the angle $\delta$ about the same as the angle between the line of sight and the line connecting the two pulsars. In turn, the latter is related to the orbital phase as $\left(90^{\circ}-\phi_{\text {orb }}\right)(\bmod 180)$. Therefore, $\delta_{*} \sim\left(90^{\circ}-\phi_{\text {orb }}\right)$ when $-90^{\circ} \leqslant \phi_{\text {orb }}<90^{\circ}$ and $\delta_{*} \sim\left(\phi_{\text {orb }}-90^{\circ}\right)$ when $90^{\circ} \leqslant \phi_{\text {orb }}<270^{\circ}$, where $\delta_{*}$ is the value of $\delta$ at the moment of the closest approach. For instance, at the orbital phase $185^{\circ}, \delta_{*} \sim 95^{\circ}$ and at $305^{\circ}, \delta_{*} \sim 145^{\circ}$. Therefore, $\delta_{*}$ varies within $\left[95^{\circ}, 145^{\circ}\right]$ and $\left[145^{\circ}, 180^{\circ}\right]$ for BP1 and $\mathrm{BP} 2$, respectively.

We calculated the deflection angle $\alpha_{\text {defl }}$ using two different methods. We used a simple analytical estimation for the simplified D61 model, while employing more complex numerical calculations for the modified TS02 model. In the D61 model, the system is characterized by three main parameters: the magnetic moment of the pulsar, the magnetic field in A's wind $\vec{B}_{w}$, and the angle between the two. Neither of the models depend on the absolute values of the pulsar and wind's magnetic fields. In both cases, only the ratio of these two fields matter. This boils

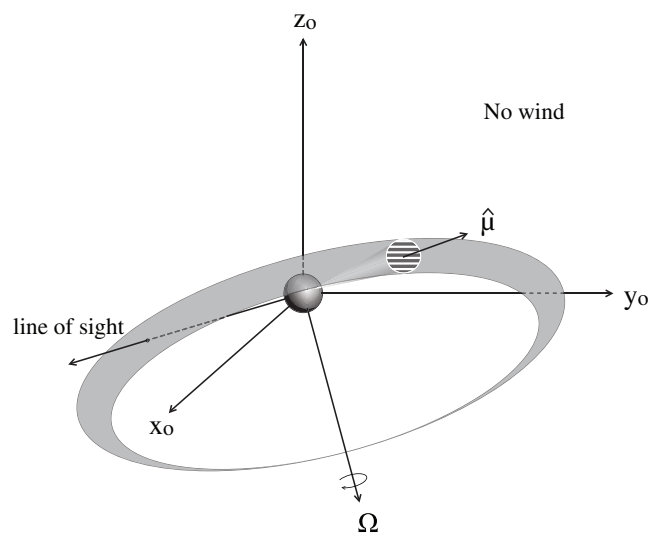

(a)

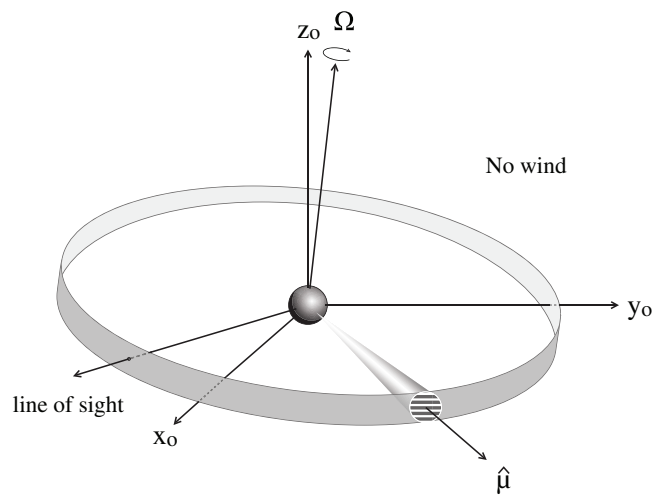

(c)

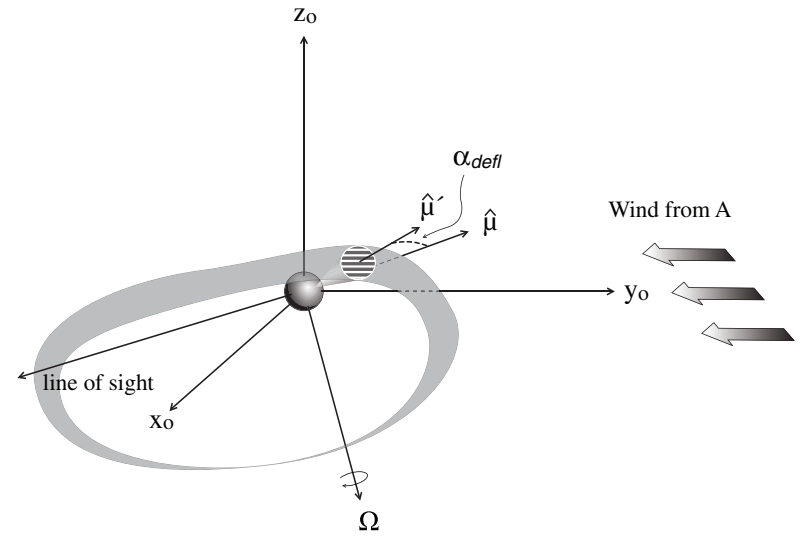

(b)

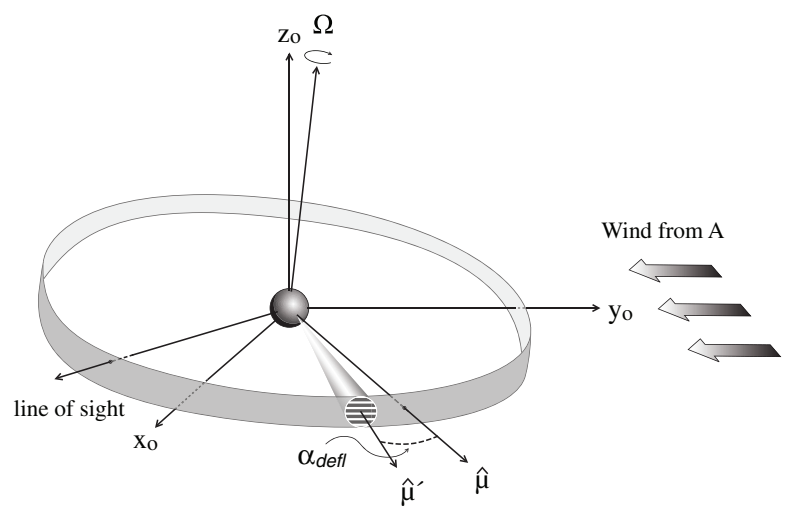

(d)

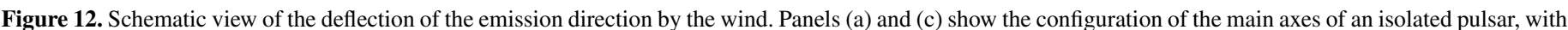

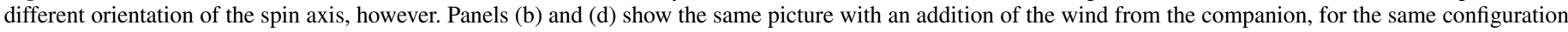

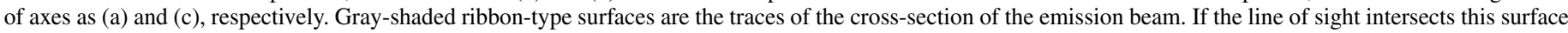

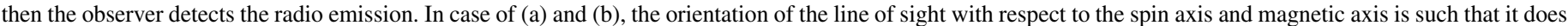

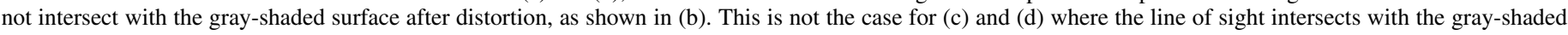
surfaces, even after distortion. 
down to the assumption that the wind from A is strongly magnetized (i.e., the shape of the boundary and stand-off distance is defined solely by the magnetic pressure balance). The pulsar wind is highly magnetized near the light cylinder. The particle component only takes over much further, closer to the termination shock. Since the wind from A reaches B after only 1000 light cylinder radii, the assumption about its high magnetization is valid. Therefore, we can describe both fields by one dimensionless parameter: stand-off distance normalized to the stellar radius. At the moment of the closest approach, the magnetic moment of B is almost aligned with the line of sight. In turn, due to the peculiarity of the double pulsar, the line of sight is nearly parallel to the orbital plane. Thus, at the moment of the closest approach, B's magnetic axis and the magnetic field of A's wind are nearly coplanar. Therefore, for approximate estimates, the full three-dimensional analysis of the system is not necessary and we only carry out the calculations for the two-dimensional configuration.

In two-dimensional polar coordinates $(r, \lambda)$, the equation for the magnetic field lines reads as

$$
\frac{d r}{B_{r}^{\mathrm{tot}}}=\frac{r d \lambda}{B_{\lambda}^{\mathrm{tot}}},
$$

where $\lambda$ is the colatitude and is equal to $90^{\circ}$ at the equator and to zero along the magnetic axis, which is the same colatitude angle that is defined in Equation (5). The angle between the local tangent to the field line and the vector $\vec{r}$ can be approximated as $r d \lambda / d r$. In order to find the deflection angle of the polar field line due to the distortions by the wind, we consider the change in $r d \lambda / d r$ :

$$
\alpha_{\mathrm{defl}}=\left(\frac{r d \lambda}{d r}\right)_{\text {distorted }}-\left(\frac{r d \lambda}{d r}\right)_{\text {undistorted }} .
$$

As a superposition of the pulsar's dipolar and wind magnetic fields we take a simple addition of the two. Therefore, it follows from Equation (22) that

$$
\alpha_{\mathrm{defl}}=\left(\frac{B_{\lambda}^{p}+B_{\lambda}^{w}}{B_{r}^{p}+B_{r}^{w}}\right)-\left(\frac{B_{\lambda}^{p}}{B_{r}^{p}}\right) .
$$

Here, $B_{r}^{p}, B_{\lambda}^{p}$ and $B_{r}^{w}, B_{\lambda}^{w}$ are $r$ and $\lambda$ components of the pulsar and wind magnetic fields, respectively. The angle between the local components of the fields can be either $\delta_{*}+\lambda+90^{\circ}$ or $90^{\circ}-\left(\delta_{*}+\lambda\right)$, depending on the orientation of the toroidal field. Thus, in the frame of the dipole, the magnetic field components can be expressed as follows:

$$
\begin{gathered}
B_{r}^{p}=-\frac{\mu_{0} m}{2 \pi} \frac{\cos \lambda}{r^{3}} \\
B_{\lambda}^{p}=\frac{\mu_{0} m}{4 \pi} \frac{\sin \lambda}{r^{3}} \\
B_{r}^{w}=\mp B_{0}^{w} \sin \left(\lambda+\delta_{*}\right) \\
B_{\lambda}^{w}=\mp B_{0}^{w} \cos \left(\lambda+\delta_{*}\right) .
\end{gathered}
$$

Here, $m$ is a magnetic moment of pulsar $\mathrm{B}$ while $B_{0}^{w}$ is a strength of the wind magnetic field. The wind magnetic field structure is believed to be toroidal. However, for the sake of simplicity, we assumed a locally uniform wind field across the whole magnetosphere of $\mathrm{B}$, which is feasible since the radius of the light cylinder is much smaller than the orbital radius. In Equations (27) and (28), $B_{r}^{w}$ and $B_{\lambda}^{w}$ can switch signs depending on whether the large-scale toroidal field of the wind is prograde with respect to the orbital motion of the pulsars or retrograde. Mathematically it is equivalent to replacing $\delta_{*}$ with $\delta_{*}+180^{\circ}$. Below, we derive the approximate expression for $\alpha_{\text {defl }}$ for the upper signs in Equations (27) and (28) and only in the end substitute $\delta_{*}+180^{\circ}$ instead of $\delta_{*}$ to account for both cases.

It is reasonable for our case $\left(\alpha_{\text {defl }} \leqslant 14.3\right)$ to limit our estimations to the field lines close to the magnetic axis, i.e., $\lambda \sim 0^{\circ}$. Then we can rewrite Equations (25)-(28) in the following way:

$$
\begin{gathered}
B_{r}^{p}=-\frac{\mu_{0} m}{2 \pi} \frac{1}{r^{3}} \\
B_{\lambda}^{p}=0 \\
B_{r}^{w}=-B_{0}^{w} \sin \delta_{*} \\
B_{\lambda}^{w}=-B_{0}^{w} \cos \delta_{*} .
\end{gathered}
$$

Here, we are only left with the upper signs in the second pair of equations. We get an approximate expression for the deflection angle by substituting Equations (29)-(32) into Equation (24)

$$
\alpha_{\mathrm{defl}}=\frac{B_{0}^{w} \cos \delta_{*}}{\frac{\mu_{0} m}{2 \pi} \frac{1}{r^{3}}+B_{0}^{w} \sin \delta_{*}} .
$$

We can rewrite Equation (33) in terms of normalized distance $\bar{r} \equiv r / r_{\mathrm{s}}$, where $r_{\mathrm{s}}=\left(\mu_{0} m / 2 \pi B_{0}^{w}\right)^{1 / 3}$ is a stand-off distance $\left(r_{\mathrm{s}} \sim 4 \times 10^{9} \mathrm{~cm}\right.$ as estimated from our boundary model),

$$
\alpha_{\mathrm{defl}}=\frac{\bar{r}^{3} \cos \delta_{*}}{1+\bar{r}^{3} \sin \delta_{*}} .
$$

From the criteria for the pulsed radio emission detectability, it follows that the values of $\bar{r}$ for which $\alpha_{\text {defl }}>14.3$ must be excluded as possible emission heights. To find such values of $\bar{r}$ for any $\delta_{*}$, we use the condition that the absolute value of the right-hand side of Equation (34) must exceed 14.3. Hence, in the case of a prograde toroidal field we have

$$
\left|\frac{\bar{r}^{3} \cos \delta_{*}}{1+\bar{r}^{3} \sin \delta_{*}}\right|>14.3 \text {. }
$$

By replacing $\delta_{*}$ with $\delta_{*}+180^{\circ}$, we get the detectability criteria for the retrograde configuration

$$
\left|\frac{-\bar{r}^{3} \cos \delta_{*}}{1-\bar{r}^{3} \sin \delta_{*}}\right|>14.3 .
$$

The minimum of the values of $\bar{r}$ that satisfy Equations (35) or (36) for any $\delta_{*}$ corresponding to BP1 and BP2 is the best upper limit we can put on the emission height with this approach. In Figure 13, we show the solutions of Equations (35) and (36), represented by the shaded areas over the contours of constant deflection angle of 14.3. As we can see in Figure 13, both orientations of the wind magnetic field produce almost the same 


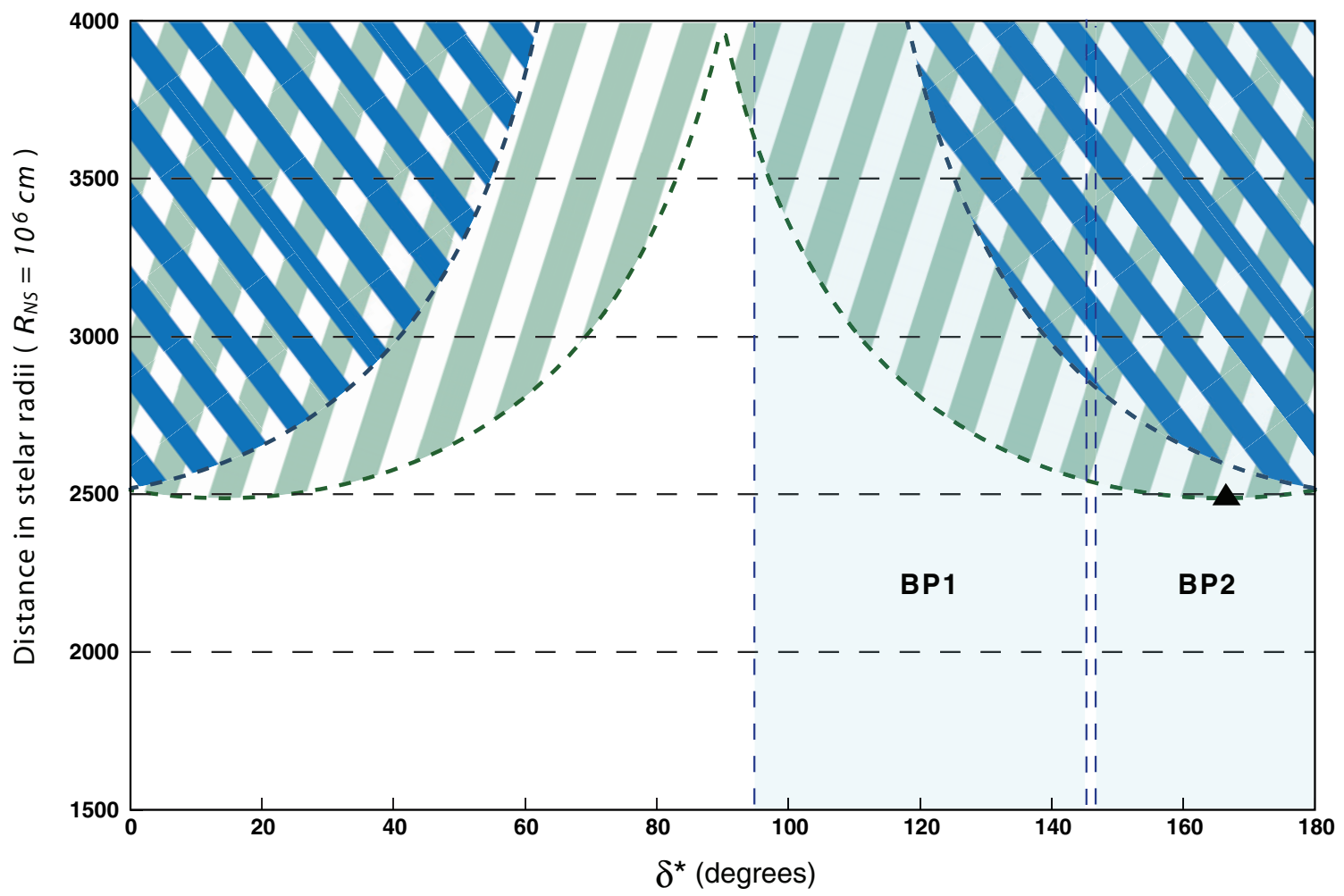

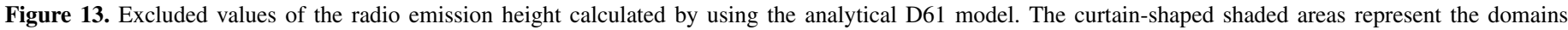

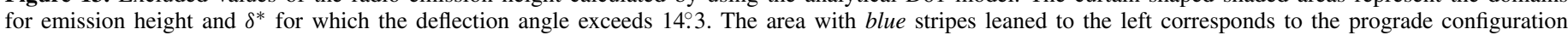

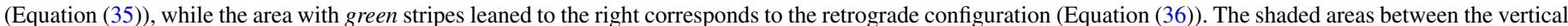

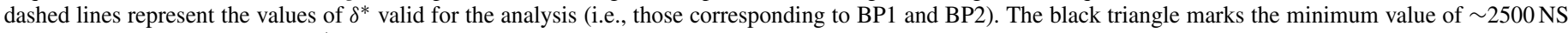
radii within the shaded range of $\delta^{*}$. Thus, $2500 \mathrm{NS}$ radii is the best upper limit for the emission height within the D61 model.

(A color version of this figure is available in the online journal.)

upper limit, which is $\sim 2500 R_{\mathrm{NS}}$. This value corresponds to $\delta_{*} \sim 170^{\circ}-180^{\circ}$, i.e., when pulsar B, pulsar A, and Earth are nearly aligned, where pulsar B is in BP2 region. This is consistent with the method by which we estimate an upper limit. At superior conjunction, the wind magnetic field is perpendicular to the magnetic axis, resulting in the largest distortion of the polar field lines. Therefore, the distance from the star surface, above which the deflection is more than 14.3, is smallest at superior conjunction.

We can use the same criteria to set an upper limit on the emission height using the numerical TS02 model. We require that the numerically calculated distortion angle of the polar field line must not exceed the angular radius of the beam $\left(\rho_{\mathrm{a}, 10} \simeq 14.3\right)$. This allows us to find the maximum emission height for each value of $\delta_{*}$.

Using the modified TSO2 code, we can trace any field line (particularly polar field lines) of B's distorted magnetosphere for any orientation of the magnetic axis with respect to A's wind. This means that we can calculate the deflection angle for any $\delta_{*}$ at any altitude. The 14.3 contour in Figure 14 shows the altitudes at which the deflection angle equals 14.3 for all values of $\delta_{*}$. The altitude with the lowest value among others is the best upper limit we can put on the emission height. Since we can only consider $\delta_{*}$ 's corresponding to $\mathrm{BP} 1$ and $\mathrm{BP} 2$, the resultant best upper limit of the emission height would be $2500 R_{\mathrm{NS}}$ for $\delta_{*} \sim 95^{\circ}$ (orbital phase of $185^{\circ}$, which is in BP1; see Figure 14).

In summary, we adapted the models of Earth's distorted magnetosphere to the double pulsar system, based on the similarities between the two. We adjusted the spin-orbital and magnetic field parameters corresponding to the observational data. Both magnetospheric models, the analytic D61 and numerical TS02, draw simplified and very extreme pictures of the double pulsar. Nevertheless, both models offer an improvement over a simple dipole and allow us to set an upper limit on the altitude of the emission region using a novel technique. Moreover, they would greatly compliment each other if somehow unified into one model.

We used the criteria of the pulsar emission detectability to estimate an upper limit of the emission height. This requires the distortion angle of the polar field line not to exceed the angular radius of the beam, derived from the observational data.

Moreover, we arrived at similar results by employing two very different models; the analytical, highly resistive Dungey type model and the numerical, fully screened modified TS02 model. Both approaches led to the conclusion that B's radio emission is generated within the inner $22 \%$ of the light cylinder.

\section{DISCUSSION}

The determination of radio pulsar emission heights is important for understanding their emission mechanisms. Pulsar B of the double pulsar system provides a unique opportunity to study different emission regions of the magnetosphere due to precession. Also, the magnetosphere is distorted, exhibiting a complicated field line structure, due to the wind of A. These distortions depend on the orbital and rotational phases of $\mathrm{B}$. Observations of these distortions, not observed in isolated pulsars, via the orbital variations of the radio intensity of B allow us to pinpoint the location of radio emission. 


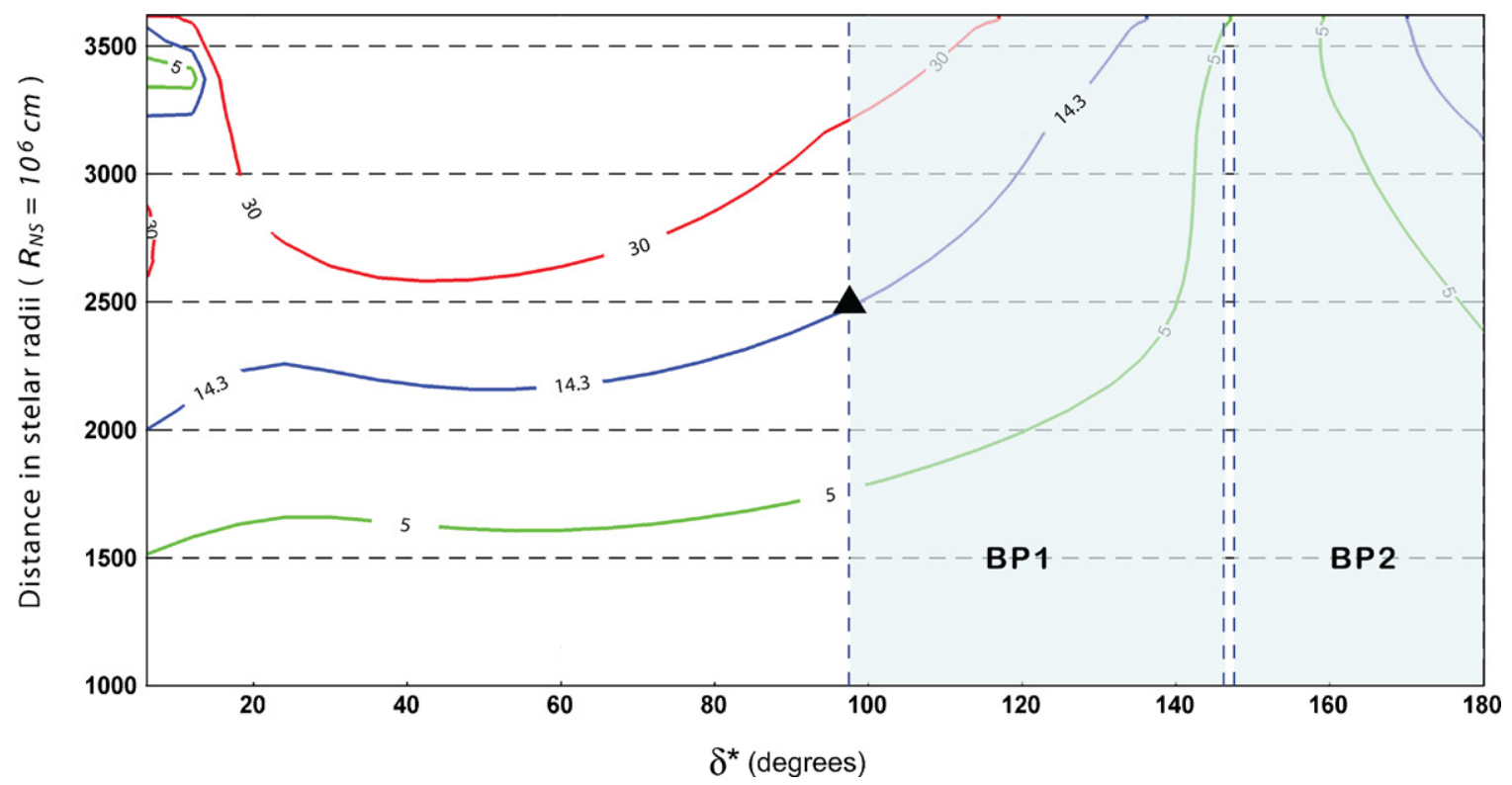

Figure 14. Fixed value contours for the deflection angle calculated by using the modified TS02 model. Each contour shows the upper limits to the emission region height for the corresponding value of the deflection angle and $\delta^{*}$. The shaded area between the vertical dashed lines represents the values of $\delta^{*}$ valid for the analysis (i.e., those corresponding to BP1 and BP2). The black triangle on the 14.3 contour marks the minimum value of 2500 NS radii within the shaded range of $\delta^{*}$. Thus, 2500 NS radii is the best upper limit for the emission height within the modified TS02 model.

(A color version of this figure is available in the online journal.)

We have applied a simple wind-magnetosphere interaction model to determine the boundary of the magnetosphere of B. The best solution describes the shape of the boundary as a polynomial, with coefficients dependent on the angle between the magnetic axis and the line connecting the two pulsars. Furthermore, the boundary was not axially symmetric, but for simplicity we modified it to be symmetric. The standoff distance ranges from $(3.8-4.5) \times 10^{9} \mathrm{~cm}$ according to the orientation of the magnetic axis with respect to the line connecting two pulsars, resulting in a size of the polynomial boundary of less than $30 \%$ of the light cylinder radius. However, this size is three times larger than the size inferred from eclipses of A. Thus, a possible explanation for this is that the particle density in the magnetosphere of B falls off significantly as a function of the radial distance from the center of the pulsar, so that the radiation of A penetrates the outer regions of B's magnetosphere.

Moreover, the variation of the boundary will change the shape of the open field line region. As a result, the spin-down luminosity of B can vary slightly due to the variation in the area of the polar cap. This causes a $1.5 \%$ periodic variation in the spin-down luminosity. It can also lead to a correction on the spin phase, but this is very small (Gourgouliatos et al. 2011). Thus, we did not consider this effect in our model.

As we determined, the range of the allowed emission height depends on the orbital motion due to the relative orientation of the magnetic axis with respect to the boundary. Also, precession changes the location of the spin axis, so that the emission height changes with time. In both of these variations, we have been calculating the emission heights for both the leading and the trailing edges of the conal elliptical beam. For a normal pulsar with its light-cylinder boundary, these two edges give the same height due to cylindrical symmetry. When the impact parameter is equal to the angular radius of the beam across the semimajor axis, we would detect a single-peak profile, resulting in one emission height. Figure 7 shows that the relative heights of emission due to the two components of the beam switch in the two bright phase regions since the orientation of the boundary changes throughout the orbit. These estimated emission heights are about $1 \%$ of the light cylinder radius or $4 \%$ of the standoff distance. Again, these should be considered lower limits if the emission does not originate on the last closed field line. Moreover, the analytical and numerical approaches to the upper limit estimate lead to the conclusion that pulsar B's radio emission is generated within $22 \%$ of the light cylinder.

In normal pulsars, radio emission heights have been calculated by using their geometry and the pulse profile widths (Kijak \& Gil 1997). These range from about 10 to $100 R_{\mathrm{NS}}$, less than $10 \%$ of the light cylinder radius. Our emission height estimates are consistent with these results. Thus, the radio emission produced by B likely has the same mechanism as for isolated pulsars, which is consistent with Lyutikov (2005). Most theories of pulsar radio emission place the generation region close to the star, typically within one stellar radius (e.g., Melrose 1995). In contrast, a model based on the anomalous cyclotronCherenkov resonance (Machabeli \& Usov 1979; Lyutikov et al. 1999) requires emission to be generated much higher up in the magnetosphere, at hundreds of stellar radii. The fairly high emission altitudes of radio emission inferred in the present paper are consistent with the latter models.

The magnetospheres of pulsars can be distorted due to rotation as proposed in Dyks \& Harding (2004), resulting in a rotational sweepback of the magnetic field lines. They found that at low altitude the rotation deflects the local direction of the magnetic field line by at most an angle of the order of $\left(r / R_{\mathrm{LC}}\right)^{2}$, where $r$ is the radial distance of the field line. We applied this rotational sweepback model to pulsar B along with our boundary model and found that the rotational sweepback is very small, because the deflection of the magnetic field line from its local direction is of order 0.1 radians. The estimated upper limit for the emission height implies that this effect is less than 0.05 radians, negligible compared with the distortions by the wind. However, this effect 
is significant when the radial distance of the field is close to the light cylinder, so that it is important in normal pulsars.

The relativistic phase-shift method can be used to determine the radio emission heights of pulsars as described in Gangadhara \& Gupta (2001) and Dyks et al. (2004). This method uses aberration and retardation effects to explain the observed pulse phase shift of pulse profiles containing core and conal components. We applied this method to the double-peaked pulse profiles of pulsar B to estimate the emission height. Because pulsar B has only a conal component, we assumed that pulse phase of zero was at the minimum between the two peaks. Then the phase shift is measured from the two peaks, leading and trailing, with respect to this reference phase. The calculation shows that the phase-shift method does not work for pulsar B. For example, the emission height on MJDs 53860 and 53939 is 6 and 23 NS radii, respectively. On MJD 54050 it is zero due to zero phase shift. Also on MJD 54400, the phase of trailing component is larger than the absolute phase of leading component, so that the emission height becomes negative. The reason of these calculated height fluctuations is that the pulse profile of $\mathrm{B}$ is not stable and varies significantly. Therefore, it is difficult to measure the shift in pulse phase accurately. Also, as there are only two peaks in the pulse profile of $\mathrm{B}$, the determination of the pulse phase zero reference point is difficult. Therefore, the measured shifts and then the emission heights may not be correct, concluding that this method cannot be used to constrain emission heights of pulsar B. However, this is a useful method of estimating emission heights of normal pulsars that have stable pulse profiles with both core and conal emission components (Gupta \& Gangadhara 2003).

In summary, by using the method presented in this paper, we can place limits on the radio emission height for any pulsar with well-determined emission geometry. The advantage of this method is that by estimating the field-line constant by tracing the magnetic field lines, we can constrain the emission heights of pulsars that have high magnetic inclinations that make them unsuitable for the other methods. Our radio emission height estimations for pulsar B will be useful for future studies and in particular can be used to constrain proposed geometrical models such as Lyutikov (2005) and Freire et al. (2009) in order to accurately explain the observations.
B.B.P.P. is supported by NRAO student observing support and M.A.M. is supported by a WV EPSCoR grant. The National Radio Astronomy Observatory is a facility of the National Science Foundation operated under cooperative agreement by Associated Universities, Inc.

\section{REFERENCES}

Barker, B. M., \& O’Connell, R. F. 1975, Phys. Rev. D, 12, 329

Breton, R. P., Kaspi, V. M., Kramer, M., et al. 2008, Science, 321, 104

Burgay, M., D’Amico, N., Possenti, A., et al. 2003, Nature, 426, 531

Clifton, T., \& Weisberg, J. M. 2008, ApJ, 679, 687

Contopoulos, I., \& Spitkovsky, A. 2006, ApJ, 643, 1139

Demorest, P., Ramachandran, R., Backer, D. C., et al. 2004, ApJ, 615, L137

Deutsch, A. J. 1955, Ann. d'Astrophys., 18, 1

Dungey, J. W. 1961, Phys. Rev. Lett., 6, 47

Dyks, J., \& Harding, A. K. 2004, ApJ, 614, 869

Dyks, J., Rudak, B., \& Harding, A. K. 2004, ApJ, 607, 939

Forbes, T. G., \& Speiser, T. W. 1971, J. Geophys. Res., 76, 7541

Freire, P. C. C., Wex, N., Kramer, M., et al. 2009, MNRAS, 396, 1764

Gangadhara, R. T. 2004, ApJ, 609, 335

Gangadhara, R. T., \& Gupta, Y. 2001, ApJ, 555, 31

Gil, J., Gronkowski, P., \& Rudnicki, W. 1984, A\&A, 132, 312

Gil, J., \& Kijak, K. 1993, A\&A, 273, 563

Gourgouliatos, K. N., Lyutikov, M., Lomiashvili, D., \& Perera, B. B. P. 2011, in AIP Conf. Ser. 1357, Radio Pulsars: An Astrophysical Key to Unlock the Secrets Of The Universe, ed. M. Burgay et al. (Melville, NY: AIP), 304

Gupta, Y., \& Gangadhara, R. T. 2003, ApJ, 584, 418

Hankins, T. H., Rankin, J. M., \& Eilek, J. A. 2009, in Astro2010: The Astronomy and Astrophysics Decadal Survey, Astro2010 Science Frontier Panel Stars and Stellar Evolution, Science White Papers, no., 112

Kijak, J., \& Gil, J. 1997, MNRAS, 288, 631

Kijak, J., \& Gil, J. 2003, ApJ, 397, 969

Kramer, M., Stairs, I. H., Manchester, R. N., et al. 2006, Science, 314, 97

Lyne, A. G., Burgay, M., Kramer, M., et al. 2004, Science, 303, 1153

Lyutikov, M. 2004, MNRAS, 353, 1095

Lyutikov, M. 2005, MNRAS, 362, 1078

Lyutikov, M., Blandford, R. D., \& Machabeli, G. 1999, MNRAS, 305, 338

Machabeli, G. Z., \& Usov, V. V. 1979, Sov. Astron. Lett., 5, 238

Melrose, D. B. 1995, J. Astrophys. Astron., 16, 137

Mitra, D., \& Rankin, J. M. 2002, ApJ, 577, 322

Perera, B. B. P., McLaughlin, M. A., Kramer, M., et al. 2010, ApJ, 721, 1193

Radhakrishnan, V., \& Cooke, D. J. 1969, Astrophys. Lett, 3, 225

Rankin, J. M. 1990, ApJ, 352, 247

Spitkovsky, A. 2006, ApJ, 648, L51

Tsyganenko, N. A. 2002a, J. Geophys. Res. (Space Phys.), 107, 1179

Tsyganenko, N. A. 2002b, J. Geophys. Res. (Space Phys.), 107, 1176

Tsyganenko, N. A., \& Sitnov, M. I. 2007, J. Geophys. Res. (Space Phys.), 112, A06225 